TRANSACTIONS OF THE

AMERICAN MATHEMATICAL SOCIETY

Volume 352, Number 12, Pages 5703-5743

S 0002-9947(00)02560-5

Article electronically published on July 6, 2000

\title{
MULTIPLE SOLUTIONS FOR QUASI-LINEAR PDES INVOLVING THE CRITICAL SOBOLEV AND HARDY EXPONENTS
}

\author{
N. GHOUSSOUB AND C. YUAN
}

ABSTRACT. We use variational methods to study the existence and multiplicity of solutions for the following quasi-linear partial differential equation:

$$
\left\{\begin{aligned}
-\triangle_{p} u & =\lambda|u|^{r-2} u+\mu \frac{|u|^{q-2}}{|x|^{s}} u \text { in } \Omega, \\
\left.u\right|_{\partial \Omega} & =0
\end{aligned}\right.
$$

where $\lambda$ and $\mu$ are two positive parameters and $\Omega$ is a smooth bounded domain in $\mathbf{R}^{n}$ containing 0 in its interior. The variational approach requires that $1<p<n, p \leq q \leq p^{*}(s) \equiv \frac{n-s}{n-p} p$ and $p \leq r \leq p^{*} \equiv p^{*}(0)=\frac{n p}{n-p}$, which we assume throughout. However, the situations differ widely with $q$ and $r$, and the interesting cases occur either at the critical Sobolev exponent $\left(r=p^{*}\right)$ or in the Hardy-critical setting $(s=p=q)$ or in the more general Hardy-Sobolev setting when $q=\frac{n-s}{n-p} p$. In these cases some compactness can be restored by establishing Palais-Smale type conditions around appropriately chosen dual sets. Many of the results are new even in the case $p=2$, especially those corresponding to singularities (i.e., when $0<s \leq p$ ).

\section{InTRODUCTION}

Consider the following quasi-linear partial differential equation:

$\left(\mathrm{P}_{\lambda, \mu}\right) \quad\left\{\begin{aligned}-\triangle_{p} u & =\lambda|u|^{r-2} u+\mu \frac{|u|^{q-2}}{|x|^{s}} u \text { in } \Omega, \\ \left.u\right|_{\partial \Omega} & =0,\end{aligned}\right.$

where $\lambda$ and $\mu$ are two positive parameters and $\Omega$ is a smooth bounded domain in $\mathbf{R}^{n}$ containing 0 in its interior. We shall assume throughout that $0 \leq s \leq p<n$.

The starting point of the variational approach to these problems is the following Sobolev-Hardy inequality, which is essentially due to Caffarelli, Kohn and Nirenberg [8]. Assume that $1<p<n$ and that $q \leq p^{*}(s) \equiv \frac{n-s}{n-p} p$; then there is a constant $C>0$ such that

$$
C\left(\int_{\Omega} \frac{|u|^{q}}{|x|^{s}}\right)^{\frac{p}{q}} d x \leq \int_{\Omega}|\nabla u|^{p} d x \quad \text { for all } u \in H_{0}^{1, p}(\Omega) .
$$

We use $\mu_{s, q}(\Omega)$ to denote the best Sobolev-Hardy constant, i.e. the largest constant $C$ satisfying the above inequality for all $u \in H_{0}^{1, p}(\Omega)$; that is,

$$
\mu_{s, q}(\Omega)=\inf _{u \in H_{0}^{1, p}(\Omega), u \neq 0} \frac{\int_{\Omega}|\nabla u|^{p} d x}{\left(\int_{\Omega} \frac{|u|^{q}}{|x|^{s}}\right)^{\frac{p}{q}} d x} .
$$

Received by the editors August 11, 1998.

2000 Mathematics Subject Classification. Primary 35J20, 35J70, 47J30, 58E30. 
In the important case where $q=p^{*}(s)$, we shall simply denote $\mu_{s, p^{*}(s)}$ as $\mu_{s}$. Note that $\mu_{0}$ is nothing but the best constant in the Sobolev inequality while $\mu_{p}$ is the best constant in the Hardy inequality, i.e.,

$$
\mu_{p}(\Omega)=\inf _{u \in H_{0}^{1, p}(\Omega), u \neq 0} \frac{\int_{\Omega}|\nabla u|^{p} d x}{\int_{\Omega} \frac{|u|^{p}}{|x|^{p}} d x} .
$$

We shall always assume that $p \leq r \leq p^{*} \equiv p^{*}(0)=\frac{n p}{n-p}$ for the non-singular term in such a way that the functional

$$
E_{\lambda, \mu}(u)=\frac{1}{p} \int_{\Omega}|\nabla u|^{p} d x-\frac{\lambda}{r} \int_{\Omega}|u|^{r} d x-\frac{\mu}{q} \int_{\Omega} \frac{|u|^{q}}{|x|^{s}} d x
$$

is then well defined on the Sobolev space $H_{0}^{1, p}(\Omega)$. The (weak) solutions of the problem $\left(P_{\lambda, \mu}\right)$ are then the critical points of the functional $E_{\lambda, \mu}$.

Another relevant parameter will be the first "eigenvalue" of the $p$-Laplacian $-\Delta_{p}$, defined as

$$
\lambda_{1}(\Omega) \equiv \mu_{0, p}(\Omega)=\inf \left\{\int_{\Omega}|\nabla w|^{p} d x: w \in H_{0}^{1, p}(\Omega), \int_{\Omega}|w|^{p} d x=1\right\} .
$$

Here are the main results of this paper.

Theorem 1.1 (Hardy-Sobolev subcritical singular and non-singular terms). Suppose $1<p \leq q<p^{*}(s)$ and $r<p^{*}$. Assume one of the following conditions holds:

(1) (High order singular term) $p<q, p \leq r, \lambda>0$ and $\mu>0$.

(2) (Low order singular term) $p=q, p<r, \lambda>0$ and $\mu_{s, p}>\mu>0$.

Then $\left(P_{\lambda, \mu}\right)$ has infinitely many solutions. Moreover, $\left(P_{\lambda, \mu}\right)$ has an everywhere positive solution with least energy and another one that is sign-changing.

Theorem 1.2 (Hardy-critical singular term). Suppose $1<p=q=p^{*}(s)$ (i.e., $s=p)$.

1. (Subcritical non-singular term) If $r<p^{*}$, then $\left(P_{\lambda, \mu}\right)$ has infinitely many solutions -at least one of them being positive- for any $\lambda>0$ and $0<\mu<\mu_{p}$.

2. (Critical non-singular term) If $r=p^{*}$ and $\Omega$ is star-shaped. Then $\left(P_{\lambda, \mu}\right)$ has no non-trivial solution for any $\lambda>0, \mu>0$.

Theorem 1.3 (Hardy-Sobolev critical singular term). Suppose $1 \leq p<q=p^{*}(s)$ (i.e., $s<p)$.

1. (High order non-singular term) Assume $p<r<p^{*}$ and $\lambda>0, \mu>0$.

- If $n>\frac{p(p-1) r+p^{2}}{p+(p-1)(r-p)}$ (in particular if $\left.n \geq p^{2}\right)$, then $\left(P_{\lambda, \mu}\right)$ has a positive solution.

- If $n>\frac{p(p-1) r+p}{1+(p-1)(r-p)}$ (in particular if $\left.n>p^{3}-p^{2}+p\right)$, then $\left(P_{\lambda, \mu}\right)$ has also a sign-changing solution.

2. (Low order non-singular term) Assume $p=r<p^{*}$ and $0<\lambda<\lambda_{1}, \mu>0$.

- If $n \geq p^{2}$, then $\left(P_{\lambda, \mu}\right)$ has a positive solution.

- If $n>p^{3}-p^{2}+p$, then $\left(P_{\lambda, \mu}\right)$ has also a sign-changing solution.

3. (Sobolev-critical non-singular term) Assume $r=p^{*}$ and $\Omega$ is star-shaped, then $\left(P_{\lambda, \mu}\right)$ has no non-trivial solution for any $\lambda>0$ and any $\mu>0$.

Theorem 1.4 (Sobolev-critical non-singular term and subcritical singular term). Suppose $1<p \leq q<p^{*}(s)$ and $r=p^{*}$. 
1. (High order singular term) Assume that $p<q$ and $\lambda>0, \mu>0$.

- If $n>\frac{p(p-1)(q-s)+p^{2}}{p+(p-1)(q-p)}$ (in particular if $\left.n \geq p^{2}-(p-1) s\right)$, then $\left(P_{\lambda, \mu}\right)$ has a positive solution.

- If $n>\frac{p(p-1)(q-s)+p}{1+(p-1)(q-p)}$ (in particular if $\left.n>p(p-1)(p-s)+p\right)$, then $\left(P_{\lambda, \mu}\right)$ has also a sign-changing solution.

2. (Low order non-singular term) Assume $p=q$ and $\lambda>0, \mu_{s, p}>\mu>0$.

- If $n \geq p^{2}-(p-1) s$, then $\left(P_{\lambda, \mu}\right)$ has a positive solution.

- If $n>p((p-1)(p-s)+1)$, then $\left(P_{\lambda, \mu}\right)$ has also a sign-changing solution.

The following tables summarize our results.

TABLE 1. Sobolev-subcritical non-singular term

\begin{tabular}{|c|c|c|c|c|}
\hline Singular term & Parameters & Non-singular term & Dimension & \# of solutions \\
\hline $\begin{array}{c}\text { (HS-subcritical) } \\
p<q<p^{*}(s) \\
p=q<p^{*}(s)\end{array}$ & $\begin{array}{c}\lambda>0 ; \mu>0 \\
\mu_{s, p}>\mu>0 ; \lambda>0\end{array}$ & $\begin{array}{c}1 \leq p \leq r<p^{*} \\
1 \leq p<r<p^{*}\end{array}$ & $\begin{array}{c}n>p \\
n>p\end{array}$ & $\begin{array}{c}\text { Infinite } \\
\text { One positive } \\
\text { One positive }\end{array}$ \\
\hline $\begin{array}{c}\text { (H-critical) } \\
p=q=p^{*}(s)\end{array}$ & $\lambda>0 ; \mu_{p}>\mu>0$ & $1 \leq p<r<p^{*}$ & $n>p$ & $\begin{array}{c}\text { Infinite } \\
\text { (One positive) }\end{array}$ \\
\hline (HS-critical) \\
$p<q=p^{*}(s)$ & $\lambda>0 ; \mu>0$ & $\begin{array}{c}1 \leq p<r<p^{*} \\
2 \leq p<r<p^{*} \\
\end{array}$ & $\begin{array}{c}n>\frac{p(p-1) r+p^{2}}{p+(p-1)(r-p)} \\
n>\frac{p(p-1) r+p}{1+(p-1)(r-p)} \\
n \geq p^{2} \\
\end{array}$ & $\begin{array}{c}\text { One positive } \\
\text { Two } \\
\end{array}$ \\
$\lambda_{1}>\lambda>0 ; \mu>0$ & $\begin{array}{c}\text { One positive } \\
\text { Two }\end{array}$ \\
\hline
\end{tabular}

TABLE 2. Sobolev-critical non-singular term

\begin{tabular}{|c|c|c|c|c|}
\hline Singular term & Parameters & Non-singular term & Dimension & \# of solutions \\
\hline $1 \leq p=q<p^{*}(s)$ & $\mu_{s, p}>\mu>0$ & $r=p^{*}$ & $n>p^{2}-(p-1) s$ & One positive \\
$2 \leq p=q<p^{*}(s)$ & and $\lambda>0$ & - & $n>p((p-1)(p-s)+1)$ & Two \\
\hline $1 \leq p<q<p^{*}(s)$ & $\lambda>0 ; \mu>0$ & $r=p^{*}$ & $n>\frac{p(p-1)(q-s)+p^{2}}{p+(p-1)(q-p)}$ & One positive \\
$2 \leq p<q<p^{*}(s)$ & - & - & $n>\frac{p(p-1)(q-s)+p}{1+(p-1)(q-p)}$ & Two \\
\hline$p \leq q=p^{*}(s)$ & $\lambda>0, \mu>0$ & $r=p^{*}$ & $n>p$ & None \\
\hline
\end{tabular}

\section{A Pohozaev-type identity}

In this section, we start by identifying the constraints on the problem of existence of solutions for $\left(P_{\lambda, \mu}\right)$. Here is the main result.

Theorem 2.1. If $\Omega$ is a star-shaped domain in $\mathbf{R}^{n}$, then problem $\left(P_{\lambda, \mu}\right)$ has no solution in the doubly critical case: That is, for $r=p^{*}$ and $q=p^{*}(s)=\frac{n-s}{n-p} p$, the problem $\left(P_{\lambda, \mu}\right)$ has no non-trivial solution.

Assume $\Omega$ is a star-shaped domain. Then, if we let $v$ denote the outwards normal to $\partial \Omega$, then $\langle x, v\rangle>0$ on $\partial \Omega$. We assume we have the necessary regularity in the following operations; otherwise, we can use an approximation argument as in Guedda and Veron [20].

Multiplying the equation $\left(P_{\lambda, \mu}\right)$ by $\langle x, \nabla u\rangle$ on both sides and integrate by parts, we get

$$
\frac{p-1}{p} \int_{\partial \Omega}|\nabla u|^{p}\langle x, v\rangle d x+\frac{n-p}{p} \int_{\Omega}|\nabla u|^{p} d x=\mu \frac{n-s}{q} \int_{\Omega} \frac{|u|^{q}}{|x|^{s}} d x+\lambda \frac{n}{r} \int_{\Omega}|u|^{r} d x .
$$


On the other hand, multiplying the equation by $u$ and integrating, we get

$$
\int_{\Omega}|\nabla u|^{p}=\mu \int_{\Omega} \frac{|u|^{q}}{|x|^{s}} d x+\lambda \int_{\Omega}|u|^{r} d x .
$$

Putting the two identities together, we have

$$
\frac{p-1}{p} \int_{\partial \Omega}|\nabla u|^{p}\langle x, v\rangle d \sigma=\mu\left(\frac{n-s}{q}-\frac{n-p}{p}\right) \int_{\Omega} \frac{|u|^{q}}{|x|^{s}} d x+\lambda\left(\frac{n}{r}-\frac{n-p}{p}\right) \int_{\Omega}|u|^{r} .
$$

So if $r=\frac{n p}{n-p}=p^{*}$ and $q=\frac{n-s}{n-p} p$, the problem has no non-trivial solution.

\section{The extremal functions in the Hardy-Sobolev inequalities}

In this section, we summarize the needed results concerning the Hardy-Sobolev inequalities. We first recall the Hardy inequality.

Lemma 3.1 ([13]). Assume that $1<p<n$ and $u \in H^{1, p}\left(\mathbf{R}^{n}\right)$. Then:

(1) $\frac{u}{|x|} \in L^{p}\left(\mathbf{R}^{n}\right)$.

(2) (Hardy Inequality) $\int_{\mathbf{R}^{n}} \frac{|u|^{p}}{|x|^{p}} d x \leq C_{n, p} \int_{\mathbf{R}^{n}}|\nabla u|^{p} d x$, where $C_{n, p}=\left(\frac{p}{n-p}\right)^{p}$.

(3) The constant $C_{n, p}$ is optimal.

The following extension of the Hardy and Sobolev inequalities is essentially due to Caffarelli, Kohn and Nirenberg[8].

Lemma 3.2 (Sobolev-Hardy Inequality). Assume that $1<p<n$ and that $p \leq$ $q \leq p^{*}(s):=\frac{n-s}{n-p} p$. Then:

(1) There exists a constant $C>0$ such that for any $u \in H_{0}^{p}(\Omega)$,

$$
\left(\int_{\Omega} \frac{|u|^{q}}{|x|^{s}}\right)^{p} d x \leq C\left(\int_{\Omega}|\nabla u|^{p}\right)^{q} d x
$$

(2) The map $u \rightarrow \frac{u}{x^{s / q}}$ from $H_{0}^{p}(\Omega)$ into $L^{q}(\Omega)$ is compact provided $q<p^{*}(s)$.

Proof. (1) For $s=0$ or $s=p$, this is just the Sobolev (resp., the Hardy) inequality. Since $p^{*}(s) \geq p$, we have $0 \leq s \leq p$. We can therefore only consider the case where $0<s<p$. By the Hardy, Sobolev and Hölder inequalities, we have

$$
\begin{aligned}
\int_{\mathbf{R}^{n}} \frac{|u|^{p^{*}(s)}}{|x|^{s}} & =\int_{\mathbf{R}^{n}} \frac{|u|^{s}}{|x|^{s}} \cdot|u|^{p^{*}(s)-s} \\
& \leq\left(\int_{\mathbf{R}^{n}} \mid \frac{|u|^{p}}{|x|^{p}}\right)^{\frac{s}{p}}\left(\int_{\mathbf{R}^{n}}|u|^{\left.\left(p^{*}(s)-s\right) \frac{p}{p-s}\right)^{\frac{p-s}{p}}}\right. \\
& =\left(\int_{\mathbf{R}^{n}} \mid \frac{|u|^{p}}{|x|^{p}}\right)^{\frac{s}{p}}\left(\int_{\mathbf{R}^{n}}|u|^{p^{*}}\right)^{\frac{p-s}{p}} \\
& \leq C_{1}\left(\int_{\mathbf{R}^{n}}|\nabla u|^{p}\right)^{\frac{s}{p}}\left(\int_{\mathbf{R}^{n}}|\nabla u|^{p}\right)^{\frac{p^{*}}{p} \cdot \frac{p-s}{p}} \\
& =C_{1}\left(\int_{\mathbf{R}^{n}}|\nabla u|^{p}\right)^{\frac{n-s}{n-p}} .
\end{aligned}
$$

Remark 3.1. If $\Omega$ is the whole space, one can show that the conditions $p \leq q=$ $p^{*}(s):=\frac{n-s}{n-p} p$ are also necessary for the above inequality to hold. Indeed, a standard scaling argument shows that $q$ must be equal to $p^{*}(s)$. On the other hand, 
if we insert into the inequality the following function ( $\rho$ and $\theta \in S^{n-1}$ being the polar coordinates),

$$
u(x)= \begin{cases}0 & \text { for }|x| \geq 1 \\ |x|^{\frac{p-n}{p}} \log \frac{1}{|x|} & \text { for } \varepsilon \leq|x|<1 \\ \varepsilon^{\frac{p-n}{p}} \log \frac{1}{\varepsilon} & \text { for }|x| \leq \varepsilon\end{cases}
$$

and

we get

$$
\frac{d u(x)}{d \rho}= \begin{cases}0, & |x| \geq 1 \\ 0, & |x| \leq \varepsilon \\ \left(1-\frac{n}{p}\right) \rho^{-\frac{n}{p}} \log \frac{1}{\rho}-\rho^{-\frac{n}{p}}, & \varepsilon \leq|x|<1\end{cases}
$$

$$
\int_{\mathbf{R}^{n}}|\nabla u|^{p} \sim \int_{\varepsilon}^{1} \rho^{-1}\left(1+\left(\frac{n}{p}-1\right) \log \frac{1}{\rho}\right)^{p} d \rho .
$$

By L'Hospital's rule, we have

$$
\lim _{\varepsilon \rightarrow 0} \frac{\int_{\varepsilon}^{1} \rho^{-1}\left(1+\left(\frac{n}{p}-1\right) \log \frac{1}{\rho}\right)^{p} d \rho}{\log ^{1+p} \frac{1}{\varepsilon}}=\frac{\frac{n}{p}-1}{1+p}
$$

and also

$$
\int_{\mathbf{R}^{n}} \frac{|u|^{q}}{|x|^{s}} \sim \int_{\varepsilon}^{1} \rho^{-s} \log ^{q} \frac{1}{\rho} \rho^{\frac{p-n}{p} q} \rho^{n-1}=\int_{\varepsilon}^{1} \frac{1}{\rho} \log ^{q} \frac{1}{\rho} \sim \log ^{1+q} \frac{1}{\varepsilon} .
$$

Thus from the inequality

we get that $q \geq p$.

$$
\log ^{1+\frac{1}{q}} \frac{1}{\varepsilon} \leq \log ^{1+\frac{1}{p}} \frac{1}{\varepsilon}
$$

The following is an extension of what is well known in the case $p=2$ and $s=0$.

Theorem 3.1. Suppose $1<p<n, 0 \leq s<p$ and $q=p^{*}(s)$. Then the following hold:

(1) $\mu_{s}(\Omega)$ is independent of $\Omega$ (and will henceforth be denoted by $\left.\mu_{s}\right)$.

(2) $\mu_{s}$ is attained when $\Omega=\mathbf{R}^{n}$ by the functions

$$
y_{a}(x)=\left(a \cdot(n-s)\left(\frac{n-p}{p-1}\right)^{p-1}\right)^{\frac{n-p}{p(p-s)}}\left(a+|x|^{\frac{p-s}{p-1}}\right)^{\frac{p-n}{p-s}}
$$

for some $a>0$. Moreover the functions $y_{a}$ are the only positive radial solutions of

in $\mathbf{R}^{n}$. Hence,

$$
-\operatorname{div}\left(|\nabla u|^{p-2} \nabla u\right)=\frac{u^{p^{*}(s)-1}}{|x|^{s}}
$$

$$
\mu_{s}\left(\int_{\mathbf{R}^{n}} \frac{\left|y_{a}\right|^{q}}{|x|^{s}}\right)^{\frac{p}{q}}=\left\|\nabla y_{a}\right\|_{p}^{p}=\int_{\mathbf{R}^{n}} \frac{\left|y_{a}\right|^{q}}{|x|^{s}}=\mu_{s}^{\frac{n-s}{p-s}} .
$$

Proof. We prove (2). We show the best constants are attained at functions

$$
u_{s}(x)=c\left(\lambda_{0}+|x|^{\frac{p-s}{p-1}}\right)^{-\frac{n-p}{p-s}} \quad(0 \leq s<p) \text {, where } \lambda_{0}>0 \text { is a constant. }
$$

For any $f$, let $f^{*}$ be its Schwarz symmetrization (or rearrangement) [21]. Then we have

$$
\int_{\mathbf{R}^{n}}\left|\nabla f^{*}\right|^{p} \leq \int_{\mathbf{R}^{n}}|\nabla f|^{p} \text { and } \int_{\mathbf{R}^{n}} \frac{\left|f^{*}\right|^{q}}{|x|^{t}} \geq \int_{\mathbf{R}^{n}} \frac{|f|^{q}}{|x|^{t}}
$$


assuming the above integrals are well defined (refer to Lieb 21], 22]). By these inequalities, we may restrict our discussion to radial symmetric functions. Thus we may consider the following variational problem:

$$
\text { Maximize } I(g)=\int_{0}^{\infty}|g(r)|{ }^{q} r^{n-s-1} d r, \quad \text { when } J(g)=\int_{0}^{\infty}\left|g^{\prime}(r)\right|^{p} r^{n-1} d r=C .
$$

where $C$ is a given constant. The Euler-Lagrange equation is

$$
\left(r^{n-1}\left|u^{\prime}(r)\right|^{p-2} u^{\prime}(r)\right)_{r}+k r^{n-s-1}|u|^{q-1}=0 .
$$

It can be easily verified that the functions

$$
u_{s}(x)=\left(\lambda+|x|^{\frac{p-s}{p-1}}\right)^{-\frac{n-p}{p-s}} \quad(0 \leq s<p)
$$

are solutions of $(*)$, where $\lambda>0$. To continue, we need the following lemma of Bliss ([1], [2]).

Lemma 3.3. Let $h(x) \geq 0$ be a measurable, real-valued function defined on $\boldsymbol{R}$ such that the integral $J_{0}=\int_{0}^{\infty} h^{p_{0}}(x) d x$ is finite and given. Set $g(x)=\int_{0}^{x} h(t) d t$. Then $I_{0}=\int_{0}^{\infty} g^{q_{0}}(x) x^{\alpha-q_{0}} d x$ attains its maximum value at the functions $h(x)=$ $\left(\lambda x^{\alpha}+1\right)^{-\frac{\alpha+1}{\alpha}}$, with $p_{0}$ and $q_{0}$ two constants satisfying $q_{0}>p_{0}>1, \alpha=\frac{q_{0}}{p_{0}}-1$, and $\lambda>0$, a real number.

By this lemma and with the change of variables $x=r^{\frac{p-n}{p-1}}$ we can deduce that $I(\cdot)$ attains its maximum at the functions

$$
u_{s}(x)=\left(\lambda+|x|^{\frac{p-s}{p-1}}\right)^{-\frac{n-p}{p-s}} \quad(0 \leq s<p) .
$$

Note that if

$$
h(x)=\left(\lambda x^{\alpha}+1\right)^{-\frac{\alpha+1}{\alpha}},
$$

then

$$
g(x)=\int_{0}^{x} h(t) d t=\left(\lambda+x^{-\alpha}\right)^{-\frac{1}{\alpha}} .
$$

And if $q=\frac{n-s}{n-p} p$, then $\alpha=\frac{q}{p}-1=\frac{p-s}{n-p}$. The theorem is thus proved.

Remark 3.2. As expected, the compactness of the embedding $u \rightarrow \frac{u}{x^{s / q}}$ in Lemma 3.2.(2) above does not hold when $q=p^{*}(s)$. Indeed, let

$$
f_{k}(x)=\left(\frac{1}{k}\right)^{\frac{n-p}{p(p-s)}}\left(\frac{1}{k}+|x|^{\frac{p-s}{p-1}}\right)^{\frac{p-n}{p-s}},
$$

and set $\left\|\nabla f_{k}(x)\right\|_{p}^{p}=A$ and $\int_{\Omega} \frac{\left|f_{k}(x)\right|^{*}(s)}{|x|^{s}} d x=C$. Let

$$
h_{k}(x)=f_{k}(x)-\left(\frac{1}{k}\right)^{\frac{n-p}{p(p-s)}}\left(\frac{1}{k}+1\right)^{\frac{p-n}{p-s}}
$$

for $|x| \leq 1$, so that $h_{k}(x) \in H_{0}^{1, p}(B)$ and $\left\|h_{k}\right\|_{H_{0}^{1, p}(\Omega)} \rightarrow A^{\frac{1}{p}}$. Hence $\left\{h_{k}\right\}$ is bounded in $H_{0}^{1, p}(\Omega)$ and $\left\|\frac{h_{k}}{|x|^{s / p^{*}(s)}}\right\|_{L^{p^{*}(s)}} \rightarrow C^{1 / p^{*}(s)}$. Now, $h_{k}(x) \rightarrow 0$ for $|x| \neq 0$ and 0 is the only possible cluster point of $\left\{\frac{h_{k}}{|x|^{s / p^{*}(s)}}\right\}$ in $L^{p^{*}(s)}(\Omega)$, which is impossible since $C \neq 0$. 


\section{The COMPaCtNess Lemmas}

This section deals with the compactness properties of the functional

$$
E_{\lambda, \mu}(u)=\frac{1}{p} \int_{\Omega}|\nabla u|^{p} d x-\frac{\lambda}{r} \int_{\Omega}|u|^{r} d x-\frac{\mu}{q} \int_{\Omega} \frac{|u|^{q}}{|x|^{s}} d x .
$$

We recall the following standard definition.

Definition 4.1. A $C^{1}$-functional $E$ on Banach space $X$ satisfies the Palais-Smale condition at the level $c$ (in short $\left.(\mathrm{PS})_{c}\right)$, if every sequence $\left(u_{n}\right)_{n}$ satisfying $\lim _{n} E\left(u_{n}\right)$ $=c$ and $\lim _{n}\left\|E^{\prime}\left(u_{n}\right)\right\|=0$ has a convergent subsequence.

Define the following function:

$$
L(\mu, s)= \begin{cases}\frac{p-s}{p(n-s)}\left(\frac{\mu_{s}^{n-s}}{\mu^{n-p}}\right)^{\frac{1}{p-s}} & \text { if } s<p \\ +\infty & \text { if } s=p \text { and } \mu<\mu_{p}, \\ 0 & \text { if } s=p \text { and } \mu \geq \mu_{p} .\end{cases}
$$

Theorem 4.1. Assume $0 \leq s \leq p<n, p \leq q \leq p^{*}(s)$ and $p \leq r \leq p^{*}$.

(1) If $p \leq q<p^{*}(s)$ and $r<p^{*}$, then for any $\lambda>0$ and any $\mu>0$, the functional $E_{\lambda, \mu}$ satisfies $(\mathrm{PS})_{c}$ for all $c$.

(2) If $p \leq q=p^{*}(s)$ and $r<p^{*}$, then for any $\lambda>0$ and any $\mu>0$, the functional $E_{\lambda, \mu}$ satisfies $(\mathrm{PS})_{c}$ for all $c<L(\mu, s)$.

(3) If $p \leq q<p^{*}(s)$ and $r=p^{*}$, then for any $\lambda>0$ and any $\mu>0$, the functional $E_{\lambda, \mu}$ satisfies $(\mathrm{PS})_{c}$ for all $c<L(\lambda, 0)=\frac{1}{n}\left(\frac{\mu_{0}^{n}}{\lambda^{n-p}}\right)^{\frac{1}{p}}$.

Note that statement 2 above yields that $E_{\lambda, \mu}$ also satisfies $(\mathrm{PS})_{c}$ for all $c$ when $p=q=q^{*}(s)$ (i.e., when $s=p$ ) as long as $\mu<\mu_{p}$. This solves a problem in [13] and [25], where only a certain singular Palais-Smale condition is established. On the other hand, when $\mu=1, p=2$ and $s=0$, we recover the (by now well known) restricted compactness properties that appears in Yamabe-type problems, [4].

We first recall a few known results.

Lemma 4.1 (25]). Let $x, y \in \mathbf{R}^{n}$, and let $\langle\cdot, \cdot\rangle_{e}$ be the standard scalar product in $\mathbf{R}^{n}$. Then

$$
\left\langle|x|^{p-2} x-|y|^{p-2} y, x-y\right\rangle_{e} \geq \begin{cases}C_{p}|x-y|^{p}, & \text { if } p \geq 2, \\ C_{p} \frac{|x-y|^{2}}{\left(|x|+\left.|y|\right|^{2-p}\right.}, & \text { if } 1<p<2 .\end{cases}
$$

The following result of Brezis and Lieb ([4]) will be useful in the sequel.

Lemma 4.2. Suppose $f_{n} \rightarrow f$ a.e. and $\left\|f_{n}\right\|_{p} \leq C<\infty$ for all $n$ and for some $0<p<\infty$. Then

$$
\lim _{n \rightarrow \infty}\left\{\left\|f_{n}\right\|_{p}^{p}-\left\|f_{n}-f\right\|_{p}^{p}\right\}=\|f\|_{p}^{p} .
$$

Lemma 4.3. Let $\left(u_{n}\right)_{n}$ be a bounded sequence in $H_{0}^{1, p}(\Omega)$ and let $\left(q_{n}\right)_{n}$ be a sequence such that $p<q_{n} \leq p^{*}(s), q_{n} \rightarrow p^{*}(s)$ as $n \rightarrow \infty$. Then there exists a subsequence (without loss of generality still denoted by $\left.\left(u_{n}\right)_{n}\right)$ such that:

(1) $u_{n} \rightarrow u$ weakly in $H_{0}^{1, p}(\Omega)$.

(2) $u_{n} \rightarrow u$ in $L^{r}(\Omega)$ if $1<r<p^{*}=\frac{n p}{n-p}$.

(3) $u_{n} \rightarrow u$ almost everywhere.

(4) $\frac{u_{n}}{x} \rightarrow \frac{u}{x}$ weakly in $L^{p}(\Omega)$. 
(5) For any $f \in H_{0}^{1, p}(\Omega)$,

(6) If $p \geq 2$, then

$$
\int_{\Omega} \frac{\left|u_{n}\right|^{q_{n}-2} u_{n}}{|x|^{s_{n}}} f \rightarrow \int_{\Omega} \frac{|u|^{p^{*}(s)-2} u}{|x|^{s}} f
$$

$$
\begin{gathered}
\int_{\Omega}\left|u_{n}\right|^{q_{n}} \leq \int_{\Omega}|u|^{q_{n}}+\int_{\Omega}\left|u_{n}-u\right|^{q_{n}}+o(1) . \\
\int_{\Omega} \frac{\left|u_{n}-u\right|^{p^{*}(s)}}{|x|^{s}}=\int_{\Omega} \frac{\left|u_{n}\right|^{p^{*}(s)}}{|x|^{s}}-\int_{\Omega} \frac{|u|^{p^{*}(s)}}{|x|^{s}}+o(1) .
\end{gathered}
$$

Proof. These are standard applications of the Hardy-Sobolev embedding theorem and the Brezis-Lieb result. We just give the proofs of (5) and (6). Without loss of generality, we may assume that $q_{n}<p^{*}(s)$. For (5), it is clear that

and that the integral

$$
\frac{\left|u_{n}\right|^{q_{n}-2} u_{n}}{|x|^{q^{\frac{q_{n}-1}{q_{n}}}}} \rightarrow \frac{|u|^{p^{*}(s)-2} u}{|x|^{s^{\frac{p^{*}(s)-1}{p^{*}(s)}}} \text { a.e. }}
$$

$$
\begin{aligned}
\int_{\Omega}\left|\frac{\left|u_{n}\right|^{q_{n}-1}}{|x|^{s\left(1-\frac{1}{p^{*}(s)}\right)}}\right|^{\frac{p^{*}(s)}{p^{*}(s)-1}} & =\int_{\Omega} \frac{\left|u_{n}\right|^{*^{*}(s) \frac{q_{n}-1}{p^{*}(s)-1}}}{|x|^{\frac{q_{n}-1}{p^{*}(s)-1}}} \cdot \frac{1}{|x|^{s\left(1-\frac{q_{n}-1}{p^{*}(s)-1}\right)}} \\
& \leq\left(\int_{\Omega} \frac{\left|u_{n}\right|^{p^{*}(s)}}{|x|^{s}}\right)^{\frac{q_{n}-1}{p^{*}(s)-1}} \cdot\left(\int_{\Omega} \frac{1}{|x|^{s}}\right)^{\frac{p^{*}(s)-q_{n}}{p^{*}(s)-1}}
\end{aligned}
$$

is uniformly bounded in $n$. Since $f /|x|^{\frac{s}{p^{*}(s)}} \in L^{p^{*}(s)}(\Omega)$ for any $f \in H_{0}^{1, p}(\Omega)$, the conclusion follows.

In order to prove (6), we need the following easy lemma.

Calculus Lemma. For every $1 \leq q \leq 3$, there exists a constant $C$ (depending on q) such that for $\alpha, \beta \in \mathbf{R}$ we have

$$
|| \alpha+\left.\beta\right|^{q}-|\alpha|^{q}-|\beta|^{q}-q \alpha \beta\left(|\alpha|^{q-2}+|\beta|^{q-2}\right) \mid \leq \begin{cases}C|\alpha||\beta|^{q-1} & \text { if }|\alpha| \geq|\beta|, \\ C|\alpha|^{q-1}|\beta| & \text { if }|\alpha| \leq|\beta| .\end{cases}
$$

For $q \geq 3$, there exists a constant $C$ (depending on $q$ ) such that for $\alpha, \beta \in \mathbf{R}$ we have

$$
|| \alpha+\left.\beta\right|^{q}-|\alpha|^{q}-|\beta|^{q}-q \alpha \beta\left(|\alpha|^{q-2}+|\beta|^{q-2}\right) \mid \leq C\left(|\alpha|^{q-2} \beta^{2}+\alpha^{2}|\beta|^{q-2}\right) .
$$

From this inequality, we can actually deduce the following more convenient result for any $q \geq 1$ :

$$
|| \alpha+\left.\beta\right|^{q}-|\alpha|^{q}-|\beta|^{q}-q \alpha \beta\left(|\alpha|^{q-2}+|\beta|^{q-2}\right) \mid \leq 2 C\left(|\alpha|^{q-1} \beta+\alpha|\beta|^{q-1}\right) .
$$

Now, back to the proof of (6). Let $w_{n}=u_{n}-u$; then $w_{n} \rightarrow 0$ weakly in $H_{0}^{1, p}(\Omega)$. By the above calculus lemma,

$$
\frac{\left|u_{n}\right|^{q_{n}}}{|x|^{s}}=\frac{\left|w_{n}+u\right|^{q_{n}}}{|x|^{s}} \leq \frac{\left|w_{n}\right|^{q_{n}}}{|x|^{s}}+\frac{|u|^{q_{n}}}{|x|^{s}}+C_{1} \frac{|u|\left|w_{n}\right|^{q_{n}-1}}{|x|^{s}}+C_{2} \frac{\left|w_{n}\right||u|^{q_{n}-1}}{|x|^{s}} .
$$

In view of (5), we only need to show that

$$
\lim _{n} \int_{\Omega} \frac{\left|w_{n}\right||u|^{q_{n}-1}}{|x|^{s}}=\lim _{n} \int_{\Omega} \frac{\left|w_{n}\right||u|^{q_{n}-2}}{|x|^{s\left(1-\frac{1}{p^{*}(s)}\right)}} \cdot \frac{|u|}{|x|^{\frac{s}{p^{*}(s)}}}=0 .
$$


For that, we check that

$$
\begin{gathered}
\int_{\Omega}\left(\frac{\left|w_{n}\right||u|^{q_{n}-2}}{|x|^{s\left(1-\frac{1}{p^{*}(s)}\right)}}\right)^{\frac{p^{*}(s)}{p^{*}(s)-1}}=\int_{\Omega} \frac{\mid w_{n} \frac{p^{p^{*}(s)}}{|x|^{p^{*}(s)-1}}}{\mid \frac{1}{p^{*}(s)-1}} \cdot \frac{|u|^{\frac{q_{n}-2}{p^{*}(s)-1} p^{*}(s)}}{|x|^{s \frac{q_{n}-2}{p^{*}(s)-1}}} \cdot \frac{1}{|x|^{s \frac{p^{*}(s)-q_{n}}{p^{*}(s)-1}}} \\
\leq\left(\int_{\Omega} \frac{\left|u_{n}\right|^{p^{*}(s)}}{|x|^{s}}\right)^{\frac{1}{p^{*}(s)-1}}\left(\int_{\Omega} \frac{|u|^{p^{*}(s)}}{|x|^{s}}\right)^{\frac{q_{n}-2}{p^{*}(s)-1}}\left(\int_{\Omega} \frac{1}{|x|^{s}}\right)^{\frac{p^{*}(s)-q_{n}}{p^{*}(s)-1}} .
\end{gathered}
$$

Hence it is uniformly bounded in $n$, and the claim follows.

Lemma 4.4. Let $E_{n}(u)=\frac{1}{p} \int_{\Omega}|\nabla u|^{p} d x-\frac{\mu}{q_{n}} \int_{\Omega} \frac{|u|^{q_{n}}}{|x|^{s}} d x-\frac{\lambda}{r} \int_{\Omega}|u|^{r} d x(\lambda>0, \mu>$ $0)$, where $q_{n}$ satisfy the conditions in the previous lemma and $1<p \leq r<p^{*}$. Assume the sequence $\left\{u_{n}\right\}$ satisfies $E_{n}\left(u_{n}\right) \rightarrow c, E_{n}^{\prime}\left(u_{n}\right) \rightarrow 0$. Then, there exists a subsequence, still denoted by $\left\{u_{n}\right\}$, such that for some $u \in H_{0}^{1, p}(\Omega)$ :

(1) $u_{n} \rightarrow u$ weakly in $u \in H_{0}^{1, p}(\Omega)$.

(2) $\nabla u_{n} \rightarrow \nabla u$ a.e.

(3) $\int_{\Omega}\left|\nabla u_{n}-\nabla u\right|^{p}=\int_{\Omega}\left|\nabla u_{n}\right|^{p}-\int_{\Omega}|\nabla u|^{p}+o(1)$.

(4) $\left|\nabla u_{m}\right|^{p-2} \nabla u_{m} \rightarrow|\nabla u|^{p-2} \nabla u$ weakly in $\left[L^{\frac{p}{p-1}}(\Omega)\right]^{n}$.

Proof. Since

$$
\lim _{n} E_{n}\left(u_{n}\right)=c \text { and } \lim _{n} E_{n}^{\prime}\left(u_{n}\right)=0
$$

and

$$
\left\langle E_{n}^{\prime}\left(u_{n}\right), u_{n}\right\rangle=\int_{\Omega}\left|\nabla u_{n}\right|^{p}-\mu \int_{\Omega} \frac{\left|u_{n}\right|^{q_{n}}}{|x|^{s}}-\lambda \int_{\Omega}\left|u_{n}\right|^{r}
$$

we have

$$
\begin{aligned}
o(1)\left(1+\left\|u_{n}\right\|\right)+p|c| \geq p E_{n}\left(u_{n}\right)-\left\langle E_{n}^{\prime}\left(u_{n}\right), u_{n}\right\rangle \\
\quad= \begin{cases}\mu\left(1-\frac{p}{q_{n}}\right) \int_{\Omega} \frac{|u|^{q_{n}}}{|x|^{s}} d x+\lambda\left(1-\frac{p}{r}\right) \int_{\Omega}\left|u_{n}\right|^{r} d x, & r>p, \\
\left(1-\frac{p}{q_{n}}\right) \int_{\Omega} \frac{|u|^{q_{n}}}{|x|^{s}} d x, & r=p .\end{cases}
\end{aligned}
$$

Since $\Omega$ is bounded, we have

$$
\int_{\Omega}\left|u_{n}\right|^{p}=\int_{\Omega} \frac{\left|u_{n}\right|^{p}}{|x|^{p s / q_{n}}} \cdot|x|^{p s / q_{n}} d x \leq M\left(\int_{\Omega} \frac{\left|u_{n}\right|^{q_{n}}}{|x|^{s}}\right)^{p / q_{n}},
$$

and

$$
\left\|\nabla u_{n}\right\|_{p}^{p}=p E_{n}\left(u_{n}\right)+\mu \frac{p}{q_{n}} \int_{\Omega} \frac{\left|u_{n}\right|^{q_{n}}}{|x|^{s}} d x+\lambda \frac{p}{r} \int_{\Omega}\left|u_{n}\right|^{r} d x .
$$

We conclude that $\left\{u_{n}\right\}$ is a bounded sequence in $H_{0}^{1, p}(\Omega)$.

We therefore can assume that $\left\{u_{n}\right\}$ satisfies all of the conclusions in Lemma 4.3. Now we use a technique initiated by Boccardo and Murat and already used by Garcia and Peral in a related context.

Define the functions

$$
\tau_{k}(s)= \begin{cases}s & \text { if }|s| \leq k \\ k s /|s| & \text { if }|s| \geq k\end{cases}
$$


We may assume also that $\tau_{k}\left(u_{n}-u\right) \rightarrow 0$ weakly in $H_{0}^{1, p}(\Omega)$ for any fixed positive $k$, since $\tau_{k}\left(u_{n}-u\right) \rightarrow 0$ a.e. and it is bounded. Then from the assumption we get

$$
\begin{aligned}
o(1)= & \left\langle E_{n}^{\prime}\left(u_{n}\right)-\left(E_{\lambda}^{p}\right)^{\prime}(u), \tau_{k}\left(u_{n}-u\right)\right\rangle+o(1) \\
= & \int_{\Omega}\left\langle\left|\nabla u_{n}\right|^{p-2} \nabla u_{n}-|\nabla u|^{p-2} \nabla u, \nabla \tau_{k}\left(u_{n}-u\right)\right\rangle_{e} \\
& -\lambda \int_{\Omega}\left(\frac{\left|u_{n}\right|^{q_{n}-2}}{|x|^{s}} u_{n}-\frac{|u|^{p^{*}(s)-2}}{|x|^{s}} u\right) \tau_{k}\left(u_{n}-u\right) .
\end{aligned}
$$

Since

$$
\frac{\left|u_{n}\right|^{q_{n}-2}}{|x|^{s}} u_{n} \rightarrow \frac{|u|^{p^{*}(s)-2}}{|x|^{s}} u
$$

in the weak star topology of $H^{-1, p^{\prime}}(\Omega)$ (by Lemma 4.3), we have

$$
\left|\int_{\Omega}\left(\frac{\left|u_{n}\right|^{q_{n}-2}}{|x|^{s}} u_{n}-\frac{|u|^{p^{*}(s)-2}}{|x|^{s}} u\right) \tau_{k}\left(u_{n}-u\right)\right| \leq C k,
$$

and

$$
\limsup _{n \rightarrow \infty} \int_{\Omega}\left\langle\left|\nabla u_{n}\right|^{p-2} \nabla u_{n}-|\nabla u|^{p-2} \nabla u, \nabla \tau_{k}\left(u_{n}-u\right)\right\rangle_{e} d x \leq C k .
$$

Let $e_{n}(x)=\left\langle\left|\nabla u_{n}\right|^{p-2} \nabla u_{n}-|\nabla u|^{p-2} \nabla u, \nabla \tau_{k}\left(u_{n}-u\right)\right\rangle_{e}$; then $e_{n}(x) \geq 0$ by Lemma 3.1 , and is uniformly bounded in $L^{1}(\Omega)$. Take $0<\theta<1$ and split $\Omega$ into

$$
S_{n}^{k}=\left\{x \in \Omega|| u_{n}-u \mid \leq k\right\}, \quad G_{n}^{k}=\left\{x \in \Omega|| u_{n}-u \mid>k\right\} .
$$

Then

$$
\begin{aligned}
\int_{\Omega} e_{n}^{\theta} d x & =\int_{S_{n}^{k}} e_{n}^{\theta} d x+\int_{G_{n}^{k}} e_{n}^{\theta} d x \\
& \leq\left(\int_{S_{n}^{k}} e_{n} d x\right)^{\theta}\left|S_{n}^{k}\right|^{1-\theta}+\left(\int_{G_{n}^{k}} e_{n} d x\right)^{\theta}\left|G_{n}^{k}\right|^{1-\theta} .
\end{aligned}
$$

Now, for fixed $k,\left|G_{n}^{k}\right| \rightarrow 0$ as $n \rightarrow \infty$, and from the uniform boundedness in $L^{1}$ we get

$$
\limsup _{n} \int_{\Omega} e_{n}^{\theta} d x \leq(C k)^{\theta}|\Omega|^{1-\theta} .
$$

Letting $k \rightarrow 0$, we get that $e_{n}^{\theta} \rightarrow 0$ strongly in $L^{1}$. By Lemma 4.1,

$$
\nabla u_{n} \rightarrow \nabla u \text { in } L^{q}
$$

for $1<q<p$. By passing to a subsequence, we have

$$
\nabla u_{n} \rightarrow \nabla u \text { a.e. }
$$

Thus (1) holds. As for (2), just apply Lemma 4.2. The proof of this lemma is thus complete.

Proof of Theorem 4.1.(1): If $p \leq q<p^{*}(s)$ and $r<p^{*}$, it is standard to show that the compactness of the Hardy-Sobolev embedding and of the Sobolev embedding imply that for any $\lambda>0$ and any $\mu>0$, the functional $E_{\lambda, \mu}$ satisfies $(\mathrm{PS})_{c}$ for all $c$. 
Proof of Theorem 4.1.(2): Recall that

$$
L(\mu, s)= \begin{cases}\frac{p-s}{p(n-s)}\left(\frac{\mu_{s}^{n-s}}{\mu^{n-p}}\right)^{\frac{1}{p-s}} & \text { if } s<p, \\ +\infty & \text { if } s=p \text { and } \mu<\mu_{s}, \\ 0 & \text { if } s=p \text { and } \mu \geq \mu_{s}\end{cases}
$$

and assume that $p \leq q=p^{*}(s)$ and $r<p^{*}$. We need to show that

$$
E_{\lambda, \mu}(u)=\frac{1}{p} \int_{\Omega}|\nabla u|^{p} d x-\frac{\mu}{p^{*}(s)} \int_{\Omega} \frac{|u|^{p^{*}(s)}}{|x|^{s}}-\frac{\lambda}{r} \int_{\Omega}|u|^{r} d x
$$

satisfies the Palais-Smale condition at any energy level less than $L(\mu, s)$.

For that, assume $\left\{u_{n}\right\}$ is a sequence in $H_{0}^{1, p}(\Omega)$ satisfying

$$
E_{\lambda, \mu}\left(u_{n}\right) \rightarrow c<L(\mu, s) \text { and } E_{\lambda, \mu}^{\prime}\left(u_{n}\right) \rightarrow 0
$$

By Lemma 4.4, we may assume that $\left\{u_{n}\right\}$ satisfies the conclusions of both Lemma 4.2 and Lemma 4.3. For any $v \in C_{0}^{\infty}(\Omega)$,

$$
\left\langle E_{\lambda, \mu}^{\prime}\left(u_{n}\right), v\right\rangle=\int_{\Omega}\left(\left\langle\left|\nabla u_{n}\right|^{p-2} \nabla u_{n}, \nabla v\right\rangle-\lambda\left|u_{n}\right|^{r-2} u_{n} v-\mu \frac{\left|u_{n}\right|^{p^{*}(s)-2} u_{n}}{|x|^{s}} v\right) d x
$$

which converges as $n \rightarrow \infty$ to

$$
0=\int_{\Omega}\left(\left\langle|\nabla u|^{p-2} \nabla u, \nabla v\right\rangle-\lambda|u|^{r-2} u v-\mu \frac{|u|^{p^{*}(s)-2} u}{|x|^{s}} v\right) d x=\left\langle E_{\lambda, \mu}^{\prime}(u), v\right\rangle .
$$

Hence $u \in H_{0}^{1, p}(\Omega)$ is a weak solution of $\left(P_{\lambda}, \mu\right)$. Choosing $v=u$, we have

and thus

$$
0=\left\langle E_{\lambda, \mu}^{\prime}(u), u\right\rangle=\int_{\Omega}\left(|\nabla u|^{p}-\lambda|u|^{r}-\mu \frac{|u|^{p^{*}(s)}}{|x|^{s}}\right) d x,
$$

$$
E_{\lambda, \mu}(u)=\lambda\left(\frac{1}{p}-\frac{1}{r}\right) \int_{\Omega}|u|^{r}+\mu\left(\frac{1}{p}-\frac{1}{p^{*}(s)}\right) \int_{\Omega} \frac{|u|^{p^{*}(s)}}{|x|^{s}} d x \geq 0 .
$$

By Lemmas 4.3 and 4.4, we have

$$
E_{\lambda, \mu}\left(u_{n}\right)=E_{\lambda, \mu}(u)+E_{0, \mu}\left(u_{n}-u\right)+o(1)
$$

and

$$
\begin{aligned}
o(1)=\left\langle E_{\lambda, \mu}^{\prime}\left(u_{n}\right), u_{n}-u\right\rangle & =\left\langle E_{\lambda, \mu}^{\prime}\left(u_{n}\right)-E_{\lambda, \mu}^{\prime}(u), u_{n}-u\right\rangle \\
& =\int_{\Omega}\left(\left|\nabla u_{n}-\nabla u\right|^{p}-\mu \frac{\left|u_{n}-u\right|^{p^{*}(s)}}{|x|^{s}}\right)+o(1) .
\end{aligned}
$$

If $s=p=p^{*}(s)$ and $\mu<\mu_{p}$, then

$$
o(1)=\int_{\Omega}\left(\left|\nabla u_{n}-\nabla u\right|^{p}-\mu \frac{\left|u_{n}-u\right|^{p}}{|x|^{p}}\right)+o(1) \leq\left(1-\frac{\mu}{\mu_{p}}\right) \int_{\Omega}\left(\left|\nabla u_{n}-\nabla u\right|^{p}+o(1) ;\right.
$$

that is, $u_{n} \rightarrow u$ strongly.

If $s<p$ (i.e., $p<p^{*}(s)$ ), we have, for large $n$,

$$
\begin{aligned}
E_{0, \mu}\left(u_{n}-u\right) & =E_{\lambda, \mu}\left(u_{n}\right)-E_{\lambda, \mu}(u)+o(1) \\
& \leq E_{\lambda, \mu}\left(u_{n}\right)+o(1) \leq c<L(\mu, s) .
\end{aligned}
$$

Thus, for such $n$,

$$
\left(\frac{1}{p}-\frac{1}{p^{*}(s)}\right)\left\|\nabla u_{n}-\nabla u\right\|^{p} \leq c<\frac{p-s}{p(n-s)} \mu_{s}^{\frac{n-s}{p-s}}\left(\frac{1}{\mu}\right)^{\frac{n-p}{p-s}} .
$$


By the Sobolev-Hardy inequality, we finally get

$$
\begin{aligned}
o(1) & =\int_{\Omega}\left(\left|\nabla u_{n}-\nabla u\right|^{p}-\mu \frac{\left|u_{n}-u\right|^{p^{*}(s)}}{|x|^{s}}\right) d x \\
& \geq \int_{\Omega}\left|\nabla u_{n}-\nabla u\right|^{p}-\mu \mu_{s}^{-\frac{p^{*}(s)}{p}}\left(\int_{\Omega}\left|\nabla u_{n}-\nabla u\right|^{p}\right)^{\frac{p^{*}(s)}{p}} \\
& =\left(\int_{\Omega}\left|\nabla u_{n}-\nabla u\right|^{p}\right)\left[1-\mu \mu_{s}^{-\frac{p^{*}(s)}{p}}\left(\int_{\Omega}\left|\nabla u_{n}-\nabla u\right|^{p}\right)^{\frac{p^{*}(s)-p}{p}}\right] \\
& \geq C \int_{\Omega}\left|\nabla u_{n}-\nabla u\right|^{p} d x .
\end{aligned}
$$

So again $u_{n} \rightarrow u$ in $H_{0}^{1, p}(\Omega)$ strongly.

Proof of Theorem 4.1.(3): Suppose now that $p \leq q<p^{*}(s)$ and $r=p^{*}$; then we have compactness in the singular term but we will be dealing with a non-singular term involving the critical Sobolev exponent. We have again

$$
\begin{aligned}
E_{0}\left(u_{n}-u\right) & =E_{\lambda, \mu}\left(u_{n}\right)-E_{\lambda, \mu}(u)+o(1) \\
& \leq E_{\lambda, \mu}\left(u_{n}\right)+o(1) \leq c<L(\lambda, 0)=\frac{1}{n} \mu_{0}^{\frac{n}{p}}\left(\frac{1}{\lambda}\right)^{\frac{n-p}{p}}
\end{aligned}
$$

Thus, for such $n$,

$$
\left(\frac{1}{p}-\frac{1}{p^{*}}\right)\left\|\nabla u_{n}-\nabla u\right\|^{p} \leq c<\frac{1}{n} \mu_{0}^{\frac{n}{p}}\left(\frac{1}{\lambda}\right)^{\frac{n-p}{p}},
$$

so that this time we get, from the Sobolev inequality,

$$
\begin{aligned}
o(1) & =\left\langle E_{\lambda, \mu}^{\prime}\left(u_{n}\right), u_{n}-u\right\rangle=\left\langle E_{\lambda, \mu}^{\prime}\left(u_{n}\right)-E_{\lambda, \mu}^{\prime}(u), u_{n}-u\right\rangle \\
& =\int_{\Omega}\left(\left|\nabla u_{n}-\nabla u\right|^{p}-\lambda \int_{\Omega}\left|u_{n}-u\right|^{p^{*}}\right)+o(1) \\
& =\left(\int_{\Omega}\left|\nabla u_{n}-\nabla u\right|^{p}\right)\left[1-\lambda \mu_{0}^{\frac{-p^{*}}{p}}\left(\int_{\Omega}\left|\nabla u_{n}-\nabla u\right|^{p}\right)^{\frac{p^{*}}{p}-1}\right]+o(1) \\
& \geq C \int_{\Omega}\left|\nabla u_{n}-\nabla u\right|^{p} d x .
\end{aligned}
$$

So again $u_{n} \rightarrow u$ in $H_{0}^{1, p}(\Omega)$ strongly.

\section{Min-maX PRinciples AND DUAl Sets Associated to $E_{\lambda, \mu}$}

For Banach spaces $X$ and $Y$, we use $C(X, Y)$ to denote the space of all continuous maps from $X$ to $Y$.

Definition 5.1. Let $X$ be a Banach space and $B$ be a closed subset of $X$. We say that a class $\mathcal{F}$ of compact subsets of $X$ is a homotopy-stable family with boundary $B$ provided that

(1) every set in $\mathcal{F}$ contains $B$, and

(2) for any set $A$ in $\mathcal{F}$ and any $\eta \in C([0,1] \times X ; X)$ satisfying $\eta(t, x)=x$ for all $(t, x)$ in $(\{0\} \times X) \cup([0,1] \times B)$ we have that $\eta(\{1\} \times A) \in \mathcal{F}$.

We say that the class $\mathcal{F}$ is $Z_{2}$-homotopy stable if all sets in $\mathcal{F}$ are symmetric and if we only require stability under odd homotopies $\eta$ (i.e., $\eta(t,-x)=-\eta(t, x)$ ). 
We say that a closed set $M$ is dual to the family $\mathcal{F}$ if

$$
M \cap B=\emptyset \text { and } M \cap A \neq \emptyset \text { for all } A \in \mathcal{F} \text {. }
$$

We shall need the following weakened version of the Palais-Smale condition.

Definition 5.2. A $C^{1}$-functional $E$ on Banach space $X$ satisfies the Palais-Smale condition at level $c$ and around the set $M$ (in short, $(\mathrm{PS})_{M, c}$ ), if every sequence $\left(u_{n}\right)_{n}$ satisfying $\lim _{n} E\left(u_{n}\right)=c, \lim _{n}\left\|E^{\prime}\left(u_{n}\right)\right\|=0$ and $\lim _{n} \operatorname{dist}\left(u_{n}, M\right)=0$ has a convergent subsequence.

The following theorem of Ghoussoub [17] will be frequently used in the sequel.

Theorem 5.1. Let $E$ be a $C^{1}$-functional on $X$ and consider a homotopy stable family $\mathcal{F}$ of compact subsets of $X$ with a closed boundary $B$. Let $M$ be a dual set to $\mathcal{F}$ such that

$$
\inf _{x \in M} E(x)=c:=c(E, \mathcal{F})=\inf _{A \in \mathcal{F}} \max _{x \in A} E(x) .
$$

If $E$ satisfies $(\mathrm{PS})_{M, c}$, then $M \cap K_{c} \neq \emptyset$, where $K_{c}$ is the set of all critical points of $E$ at level $c$.

If $\mathcal{F}$ is only $Z_{2}$-homotopy stable, then the result still holds true as long as the functional $E$ is even and the dual set $M$ is symmetric.

Note that the above theorem includes the classical min-max principle which holds under the assumption that $\sup _{x \in B} E(x)<c$. It is enough to notice that in that case $M=\{x \in X ; E(x) \geq c\}$ is a dual set.

Consider again the functional

$$
E_{\lambda, \mu}(u)=\frac{1}{p} \int_{\Omega}|\nabla u|^{p} d x-\frac{\lambda}{r} \int_{\Omega}|u|^{r} d x-\frac{\mu}{q} \int_{\Omega} \frac{|u|^{q}}{|x|^{s}} d x .
$$

Recall that we assume that $1<p<n, 0 \leq s \leq p, 0 \leq q \leq p^{*}(s) \equiv \frac{n-s}{n-p} p$ and that $p \leq r \leq p^{*} \equiv \frac{n p}{n-p}$, so that $E$ is a $C^{1}$-functional on the Sobolev space $H_{0}^{1, p}(\Omega)$.

A first dual set: Define the Mountain Pass class to be

$$
\mathcal{F}_{1}=\left\{\gamma \in C\left([0,1] ; H_{0}^{1, p}(\Omega)\right) ; \gamma(0)=0, \gamma(1) \neq 0 \text { and } E(\gamma(1)) \leq 0\right\},
$$

which is clearly homotopy-stable with boundary $B=\{E \leq 0\}$. Let

$$
M_{1}=\left\{u \in H_{0}^{1, p}(\Omega) ; u \neq 0,\left\langle E^{\prime}(u), u\right\rangle=0\right\} .
$$

The following Nehari-type duality property is by now standard.

Theorem 5.2. Assume $p \leq q \leq p^{*}(s)$ and one of the following cases:

(1) $p=r, p<q$ and $0<\lambda<\lambda_{1}, \mu>0$.

(2) $p<r \leq p^{*}, p=q$ and $0<\mu<\mu_{s, p}, \lambda>0$.

(3) $p<r \leq p^{*}, p<q$ and $\mu>0, \lambda>0$.

The set $M_{1}$ is then closed, is dual to $\mathcal{F}_{1}$ and satisfies

$$
\inf _{M_{1}} E_{\lambda, \mu}=c_{1}:=c\left(E_{\lambda, \mu}, \mathcal{F}_{1}\right)
$$

Proof. By definition,

$$
\left\langle E_{\lambda, \mu}^{\prime}(u), u\right\rangle=\int_{\Omega}\left(|\nabla u|^{p}-\lambda|u|^{r}-\mu \frac{|u|^{q}}{|x|^{s}}\right) d x .
$$


Note that $B \cap M_{1}=\emptyset$, since for every $u \in M_{1}$ we have

$$
E_{\lambda, \mu}(u)=\lambda\left(\frac{1}{p}-\frac{1}{r}\right) \int_{\Omega}|u|^{r} d x+\mu\left(\frac{1}{p}-\frac{1}{q}\right) \int_{\Omega} \frac{|u|^{q}}{|x|^{s}} d x>0,
$$

under the assumption that either $r$ or $q$ is different from $p$. We also show that under this assumption, we have the estimate $c_{1} \leq \inf _{u \in M_{1}} E_{\lambda, \mu}(u)$.

Let $u \neq 0$ in $M_{1}$, and consider the straight path $\gamma(t)=t u$. We have

$$
E_{\lambda, \mu}(t u)=\frac{t^{p}}{p} \int_{\Omega}|\nabla u|^{p}-\frac{\lambda t^{r}}{r} \int_{\Omega}|u|^{r}-\mu \frac{t^{q}}{q} \int_{\Omega} \frac{|u|^{q}}{|x|^{s}} .
$$

Since $\lim _{t \rightarrow \infty} E_{\lambda, \mu}(t u)=-\infty$, we have that $c_{1} \leq \sup _{0 \leq t<\infty} E_{\lambda, \mu}(t u)=E_{\lambda}\left(t_{0} u\right)$. From

$$
\frac{d E_{\lambda, \mu}(t u)}{d t}=t^{p-1} \int_{\Omega}|\nabla u|^{p}-\lambda t^{r-1} \int_{\Omega}|u|^{r}-\mu t^{q-1} \int_{\Omega} \frac{|u|^{q}}{|x|^{s}}
$$

and $\frac{d E_{\lambda, \mu}(t u)}{d t}\left(t_{0}\right)=0$, we get

$$
\int_{\Omega}|\nabla u|^{p}=t_{0}^{r-p} \cdot \lambda \int_{\Omega}|u|^{r}+\mu t_{0}^{q-p} \int_{\Omega} \frac{|u|^{q}}{|x|^{s}} .
$$

Since $u \in M_{1}$, we should have

$$
\int_{\Omega}|\nabla u|^{p}=\lambda \int_{\Omega}|u|^{r}+\mu \int_{\Omega} \frac{|u|^{q}}{|x|^{s}} .
$$

Thus, $t_{0}$ must be equal to 1 as long as either $r$ or $q$ is distinct from $p$. This clearly shows that under any of the 3 conditions above, we have

$$
c_{1} \leq \inf _{u \in M_{1}} E_{\lambda, \mu}(u)
$$

For the rest, we have to distinguish the 3 cases.

Case (1). $2 \leq p=r, p<q$ and $0<\lambda<\lambda_{1}$.

To prove that $M_{1}$ is closed, use the Sobolev-Hardy inequality and the definition of $\lambda_{1}$ to find a constant $c>0$ such that

$$
\begin{aligned}
\left\langle E_{\lambda, \mu}^{\prime}(u), u\right\rangle & =\left(1-\frac{\lambda}{\lambda_{1}}\right)\|u\|_{H_{0}^{1, p}(\Omega)}^{p}-c\|u\|_{H_{0}^{1, p}(\Omega)}^{q} \\
& =\|u\|_{H_{0}^{1, p}(\Omega)}^{p}\left(1-\frac{\lambda}{\lambda_{1}}-c\|u\|_{H_{0}^{1, p}(\Omega)}^{q-p}\right) .
\end{aligned}
$$

Choose some $\beta>0$ such that if $\|u\|<\beta$, then $1-\frac{\lambda}{\lambda_{1}}-c\|u\|_{H_{0}^{1, p}(\Omega)}^{q-p}>0$. This means that we can find some constant $\beta>0$ such that for any $u \in M_{1}$, we have $\|u\| \geq \beta$. So $M_{1}$ is closed.

To prove the intersection property, fix $\gamma \in \mathcal{F}_{1}$ joining 0 to $v$, where $v \neq 0$ and $E_{\lambda, \mu}(v) \leq 0$. Note that since $\lambda<\lambda_{1}$, we have $\left\langle E_{\lambda, \mu}^{\prime}(\gamma(t)), \gamma(t)\right\rangle>0$ for $t$ close to 0 (same proof as for the closedness of $M_{1}$ ). On the other hand, since $v \neq 0$, we have

$$
\left\langle E_{\lambda, \mu}^{\prime}(v), v\right\rangle<p E_{\lambda, \mu}(v) \leq 0 .
$$

It follows from the intermediate value theorem that there exists $t_{0}$ such that $\gamma\left(t_{0}\right) \in$ $M_{1}$. This proves the duality, and consequently $c_{1} \geq \inf \left\{E_{\lambda, \mu}(u): u \in M_{1}\right\}$.

Case (2). $1<p<r \leq p^{*}, p=q \leq p^{*}(s)$ and $0<\mu<\mu_{s, q}$. 
To prove that $M_{1}$ is closed, use the Sobolev-Hardy inequality with its best constant $\mu_{s, p}$ and the Sobolev inequality to get

$$
\begin{aligned}
\left\langle E_{\lambda, \mu}^{\prime}(u), u\right\rangle & =\int_{\Omega}|\nabla u|^{p}-\lambda \int_{\Omega}|u|^{r}-\mu \int_{\Omega} \frac{|u|^{q}}{|x|^{s}} \\
& \geq \int_{\Omega}|\nabla u|^{p}-c\left(\int_{\Omega}|\nabla u|^{p}\right)^{\frac{r}{p}}-\frac{\mu}{\mu_{s, q}} \int_{\Omega}|\nabla u|^{p} \\
& =\left(1-\frac{\mu}{\mu_{s, q}}\right)\|u\|_{H_{0}^{1, p}(\Omega)}^{p}-c\|u\|_{H_{0}^{1, p}(\Omega)}^{r} \\
& =\|u\|_{H_{0}^{1, p}(\Omega)}^{p}\left(1-\frac{\mu}{\mu_{s, q}}-c\|u\|_{H_{0}^{1, p}(\Omega)}^{r-p}\right) .
\end{aligned}
$$

Choose some $\beta>0$ such that if $\|u\|<\beta$, then $1-\frac{\mu}{\mu_{s, q}}-c\|u\|_{H_{0}^{1, p}(\Omega)}^{r-p}>0$. This means that we can find some constant $\beta>0$ such that for any $u \in M_{1}$, we have $\|u\| \geq \beta$. So $M_{1}$ is closed.

To prove the intersection property, fix $\gamma \in \mathcal{F}_{1}$ joining 0 to $v$, where $v \neq 0$ and $E_{\lambda, \mu}(v) \leq 0$. Note that since $\mu<\mu_{s, q}$, we have $\left\langle E_{\lambda, \mu}^{\prime}(\gamma(t)), \gamma(t)\right\rangle>0$ for $t$ close to 0 (same proof as for the closedness of $M_{1}$ ). On the other hand, since $v \neq 0$, we have $\left\langle E_{\lambda, \mu}^{\prime}(v), v\right\rangle<p E_{\lambda, \mu}(v) \leq 0$. It follows from the intermediate value theorem that there exists $t_{0}$ such that $\gamma\left(t_{0}\right) \in M_{1}$. This proves the duality, and consequently $c_{1} \geq \inf \left\{E_{\lambda, \mu}(u): u \in M_{1}\right\}$.

Case (3). $2 \leq p<r \leq p^{*}$ and $\lambda>0$.

To prove that $M_{1}$ is closed, again use the Sobolev-Hardy inequality and the Sobolev embedding to find constants $c^{\prime}>0, c^{\prime \prime}>0$ such that

$$
\begin{aligned}
\left\langle E_{\lambda, \mu}^{\prime}(u), u\right\rangle & \geq\|u\|^{p}-c^{\prime} \lambda\|u\|^{r}-c^{\prime \prime}\|u\|^{q} \\
& =\|u\|^{p}\left(1-c^{\prime} \lambda\|u\|^{r-p}-c^{\prime \prime}\|u\|^{q-p}\right) .
\end{aligned}
$$

Since both $r$ and $q$ are distinct from $p$, we may choose $\gamma>0$ such that for any $u \in H_{0}^{p, 1}(\Omega)$ with $\|u\|<\gamma$, we have $1-c_{1} \lambda\|u\|^{r-p}-c_{2}\|u\|^{q-p}>0$. This means that $\|u\| \geq \gamma$ for any $u \in M_{1}$; hence $M_{1}$ is closed.

For the intersection property, consider any $\gamma \in \mathcal{F}_{1}$ joining 0 and $v$. Since $p<r$, again the proof above of the closedness of $M_{1}$ yields that $\left\langle E_{\lambda, \mu}(\gamma(t)), \gamma(t)\right\rangle>0$ for $t$ close to 0 . Also since $v \neq 0$, we have

$$
\left\langle E_{\lambda, \mu}^{\prime}(v), v\right\rangle<p E_{\lambda, \mu}(v) \neq 0 .
$$

Then again, by the intermediate value theorem, we conclude that there exists $t_{0}$ such that $\gamma\left(t_{0}\right) \in M_{1}$. This proves the duality and the inequality

$$
c_{1} \geq \inf \left\{E_{\lambda, \mu}(u), u \in M_{1}\right\} .
$$

Another dual set: Denote by $S_{\rho}$ the sphere $S_{\rho}=\left\{u \in H_{0}^{1, p}(\Omega) ;\|u\|_{H_{0}^{1, p}(\Omega)}=\rho\right\}$ and by $\mathcal{H}$ the set

$$
\mathcal{H}=\left\{h: H_{0}^{1, p}(\Omega) \rightarrow H_{0}^{1, p}(\Omega) \text { an odd homeomorphism }\right\} .
$$

Let $\gamma_{Z_{2}}$ denote the Krasnoselskii genus, defined for every closed symmetric subset $D$ of $H_{0}^{1, p}(\Omega)$ as

$\gamma_{Z_{2}}(D)=\inf \left\{n\right.$; there exists an odd and continuous map $\left.h: D \rightarrow \mathbf{R}^{n} \backslash\{0\}\right\}$, 
and consider the class

$$
\mathcal{F}_{2}=\left\{A ; A \text { closed symmetric with } \gamma_{Z_{2}}\left(h(A) \cap S_{\rho}\right) \geq 2, \forall h \in \mathcal{H}\right\} .
$$

It is easy to verify that $\mathcal{F}_{2}$ is a $Z_{2}$-homotopy stable class. Let

$$
c_{2}=\inf _{A \in \mathcal{F}_{2}} \sup _{A} E_{\lambda, \mu}
$$

We shall now consider an appropriate dual set to $\mathcal{F}_{2}$. First, we recall a few facts about the following weighted eigenvalue problem $(1<p<\infty)$ :

$$
\left\{\begin{array}{l}
-\triangle_{p} u=\lambda b(x)|u|^{p-2} u \\
u \in H_{0}^{1, p}(\Omega), \quad u \neq 0
\end{array}\right.
$$

We will say that $\lambda \in \mathbf{R}$ is the eigenvalue and $u \in H_{0}^{1, p}(\Omega), u \neq 0$, is the corresponding eigenfunction of the above problem if the equality

$$
\int_{\Omega}|\nabla u|^{p-2} \nabla u \nabla \varphi d x=\lambda \int_{\Omega} b(x)|u|^{p-2} u \varphi d x
$$

holds for any $\varphi \in H_{0}^{1, p}(\Omega)$. The following lemma is well known.

Lemma 5.1 (25] [11). Assume $b(x) \geq 0, b(x) \in L^{t}(\Omega)$, and $|\{x \in \Omega: b(x)>0\}|$ $\neq 0$, where $t \geq 1$ if $p>n, t>1$ if $p=n$ and $t>\frac{n}{p}>1$ otherwise. Let $\lambda_{0}=\inf \left\{\int_{\Omega}|\nabla v|^{p} ; \int_{\Omega} b(x)|v|^{p}=1\right\}$. Then:

(1) $\lambda_{0}>0$ is the first eigenvalue of the problem $(*)$.

(2) $\lambda_{0}$ is simple, and there exists precisely one pair of normalized eigenfunctions corresponding to $\lambda_{0}$ which do not change sign in $\Omega$. Here, $v$ being normalized means that $\int_{\Omega} b(x)|v|^{p}=1$.

We use the lemma to prove the following fact:

Lemma 5.2. For $2 \leq p \leq r<p^{*}, p \leq q<p^{*}(s), \lambda>0, \mu>0$ and any $u \in$ $H_{0}^{1, p}(\Omega), u \neq 0$, there exists a unique $v=v(u) \in H_{0}^{1, p}(\Omega)$ such that

(a) $\int_{\Omega}\left(\lambda|u|^{r-p}+\mu \frac{|u|^{q-p}}{|x|^{s}}\right) v^{p}=1, v \geq 0$;

(b) $\|\nabla v\|_{p}^{p}=\inf \left\{\|\nabla \omega\|_{p}^{p}: \int_{\Omega}\left(\lambda|u|^{r-p}+\mu \frac{|u|^{q-p}}{|x|^{s}}\right)|\omega|^{p}=1\right\}$.

Furthermore, the map $u \rightarrow v(u)$ is continuous from $L^{r}(\Omega) \rightarrow H_{0}^{1, p}(\Omega)$.

Remark 5.1. It is quite unfortunate that the above lemma is not applicable -unless $p=2$ - whenever $r=p^{*}$ or when $q=p^{*}(s)$. This will create additional complications in the search for a second solution of the critical problems.

Proof. Since $\frac{n p^{*}}{s p^{*}+(q-p) n}>\frac{n}{p}$, choose $\frac{n}{p}<t<\frac{n p^{*}}{s p^{*}+(q-p) n}$; then $s t \frac{p^{*}}{p^{*}-(q-p) t}<n$. Because

$$
\int_{\Omega} \frac{|u|^{(q-p) t}}{|x|^{s t}} \leq\left(\int_{\Omega}|u|^{p^{*}}\right)^{\frac{t(q-p)}{p^{*}}}\left(\int_{\Omega} \frac{1}{|x|^{s t \cdot \frac{p^{*}}{p^{*}-(q-p) t}}}\right)^{\frac{p^{*}-(q-p) t}{p^{*}}}
$$

we get that $|u|^{q-p} /|x|^{s} \in L^{t}(\Omega)$.

The functional $\psi(u)=\|u\|^{p}=\int_{\Omega}|\nabla u|^{p} d x$ is clearly weakly lower semicontinuous and coercive. Moreover, the constraint set

$$
C=\left\{\omega \in H: \int_{\Omega}\left(\lambda|u|^{r-p}+\mu \frac{|u|^{q-p}}{|x|^{s}}\right)|\omega|^{p} d x=1\right\}
$$


is weakly closed in $H_{0}^{1, p}(\Omega)$ and $\psi(\cdot)$ is bounded below on $C$. Therefore, by the direct methods of the calculus of variations (Struwe [26], p. 4), the infimum in (b) is achieved and this infimum is the first eigenvalue of $(*)$ and thus is simple. Any function where such an infimum is achieved is the eigenfunction corresponding to the first eigenvalue of $(*)$. By Lemma 5.1, it cannot changes sign in $\Omega$. This gives the uniqueness of $v(u)$ and therefore its continuity for non-zero $u$.

Note that $\left(\nu_{1}(u), v(u)\right)$ corresponds to the first eigenpair of the (weighted) eigenvalue problem

$$
\left\{\begin{aligned}
-\triangle_{p} v & =\nu\left(\lambda|u|^{r-p}+\mu \frac{|u|^{q-p}}{|x|^{s}}\right)|v|^{p-2} v \quad \text { in } \Omega \\
v & =0 \text { on } \partial \Omega .
\end{aligned}\right.
$$

Now let

$$
M_{2}=M_{1} \cap\left\{u \in H_{0}^{1, p}(\Omega) ; \int_{\Omega}\left(\lambda|u|^{r-p}+\mu \frac{|u|^{q-p}}{|x|^{s}}\right) v(u)^{p-1} u=0\right\} .
$$

The following duality result was first noticed by G. Tarantello [28] in the case when $s=0$ and $p=2$.

Theorem 5.3. Assume $p \leq q<p^{*}(s)$ and $r<p^{*}$. Then $M_{2}$ is a closed set that is dual to $\mathcal{F}_{2}$, and

$$
\inf _{M_{2}} E_{\lambda, \mu}=c_{2}:=c\left(E_{\lambda, \mu}, \mathcal{F}_{2}\right)
$$

as long as we are in one of the following cases:

(1) $p=r, p<q$ and $0<\lambda<\lambda_{1}, 0<\mu$.

(2) $p<r, p=q$ and $0<\mu<\mu_{s, p}, 0<\lambda$.

(3) $p<r, p<q$ and $0<\mu, 0<\lambda$.

Proof. In the 3 cases, we get from Theorem 5.2 (and its proof) that $M_{1}$ is closed and that for any $u \neq 0$, there exists a unique $t(u)>0$ such that $t(u) u \in M_{1}$. Clearly, $t(u)=t(|u|)=t(-u)$ and

$$
E_{\lambda, \mu}(t(u) u)=\max _{t \geq 0} E_{\lambda, \mu}(t u) .
$$

The uniqueness of $t(u)$ and its properties tell us that the map $u \rightarrow t(u)$ is continuous on $H_{0}^{1, p}(\Omega)$ and that the map $u \rightarrow t(u) u$ defines an odd homeomorphism between $S_{\rho}$ and $M_{1}$ which gives that $\gamma_{Z_{2}}\left(A \cap M_{1}\right) \geq 2$ for all $A \in \mathcal{F}_{2}$.

On the other hand, the map $h: A \cap M_{1} \rightarrow \mathbf{R}$ given by

$$
h(u)=\int_{\Omega}\left(\lambda|u|^{r-p}+\mu \frac{|u|^{q-p}}{|x|^{s}}\right) v(u)^{p-1} u d x
$$

defines an odd and continuous map. Since $\gamma_{Z_{2}}\left(h\left(A \cap M_{1}\right)\right) \geq 2$, we get that $0 \in$ $h\left(A \cap M_{1}\right)$ which means that $A \cap M_{2} \neq \emptyset$ and $M_{2}$ is dual to $\mathcal{F}_{2}$. In particular, $c_{2} \geq \inf _{u \in M_{2}} E_{\lambda, \mu}(u)$.

To prove the reverse inequality, take $u \in M_{2}$ and let $v(u)$ be such that

$$
\int_{\Omega}\left(\lambda|u|^{r-p}+\mu \frac{|u|^{q-p}}{|x|^{s}}\right) v(u)^{p-1} u d x=0 .
$$


Let $\omega(u)$ be a minimizer for the problem:

$$
\begin{aligned}
\mu_{2}=\inf \left\{\psi(\omega) ; \omega \in H_{0}^{1, p},\right. & \int_{\Omega}\left(\lambda|u|^{r-p}+\mu \frac{|u|^{q-p}}{|x|^{s}}\right) v(u)^{p-1} \omega=0, \\
& \left.\int_{\Omega}\left(\lambda|u|^{r-p}+\mu \frac{|u|^{q-p}}{|x|^{s}}\right)|\omega|^{p}=1\right\} .
\end{aligned}
$$

Since $u \in M_{1}$, we obtain

$$
\mu_{2} \leq \frac{\|\nabla u\|_{p}^{p}}{\int_{\Omega}\left(\lambda|u|^{r}+\mu \frac{|u|^{q}}{|x|^{s}}\right)}=1 .
$$

Define $A=\operatorname{span}\{v(u), \omega(u)\} \in \mathcal{F}_{2}$. Then, clearly,

$$
1 \geq \mu_{2} \geq \frac{\|\nabla \omega\|_{p}^{p}}{\int_{\Omega}\left(\lambda|u|^{r-p}+\mu \frac{|u|^{q-p}}{|x|^{s}}\right)|\omega|^{p}}, \forall \omega \in A, \omega \neq 0 .
$$

For $\omega_{0} \in A$ satisfying $E_{\lambda, \mu}\left(\omega_{0}\right)=\sup _{A} E_{\lambda, \mu} \geq c_{2}$, we have $\omega_{0} \neq 0$ and $\omega_{0} \in M_{1}$. From the above inequality, we derive

$$
\int_{\Omega}\left(\lambda|u|^{r-p}+\mu \frac{|u|^{q-p}}{|x|^{s}}\right)\left|\omega_{0}\right|^{p} \geq\left\|\nabla \omega_{0}\right\|_{p}^{p}
$$

This implies

$$
\frac{1}{p} \int_{\Omega}\left(\lambda|u|^{r-p}+\mu \frac{|u|^{q-p}}{|x|^{s}}\right)\left(\left|\omega_{0}\right|^{p}-|u|^{p}\right) \geq \frac{\left\|\nabla \omega_{0}\right\|_{p}^{p}}{p}-\frac{\|\nabla u\|_{p}^{p}}{p} .
$$

Applying the inequality (valid for $t \geq p$ and $x, y \in \mathbf{R}$ )

$$
\frac{1}{t}\left(|x|^{t}-|y|^{t}\right) \geq \frac{1}{p}\left(|x|^{p}-|y|^{p}\right)|y|^{t-p}
$$

with $t=r($ resp. $t=q)$, we conclude that

$$
\frac{\lambda}{r} \int_{\Omega}\left|\omega_{0}\right|^{r}+\mu \frac{1}{q} \int_{\Omega} \frac{\left|\omega_{0}\right|^{q}}{|x|^{s}}-\frac{\lambda}{r} \int_{\Omega}|u|^{r}-\mu \frac{1}{q} \int_{\Omega} \frac{|u|^{q}}{|x|^{s}} \geq \frac{\left\|\nabla \omega_{0}\right\|_{p}^{p}}{p}-\frac{\|\nabla u\|_{p}^{p}}{p},
$$

that is,

$$
E_{\lambda, \mu}(u) \geq E_{\lambda, \mu}\left(\omega_{0}\right) \geq c_{2} .
$$

This finishes the proof of the theorem.

\section{The solutions in the CASE OF AN HS-SubCRitical Singular term}

In this section, we consider the problem

$$
\left(P_{\lambda, \mu}\right) \quad\left\{\begin{aligned}
-\triangle_{p} u & =\lambda|u|^{r-2} u+\frac{|u|^{q-2}}{|x|^{s}} u \text { in } \Omega, \\
\left.u\right|_{\partial \Omega} & =0,
\end{aligned}\right.
$$

where $0 \leq s \leq p<n$, in the presence of a subcritical singular term $(1<p \leq q<$ $\left.p^{*}(s)\right)$ and a subcritical non-singular term $\left(1<p \leq r<p^{*}\right)$.

Theorem 6.1 (Hardy-Sobolev subcritical singular term). Suppose $1<p \leq q<$ $p^{*}(s)$ and $r<p^{*}$. Also assume one of the following conditions:

(1) $p<q, p \leq r$ and $\lambda>0, \mu>0$.

(2) $p=q, p<r$ and $\lambda>0, \mu_{s, p}>\mu>0$. 
Then the equation $\left(\mathrm{P}_{\lambda, \mu}\right)$ has infinitely many solutions. Moreover, it has an everywhere positive solution $u_{1}$ with minimal energy and a sign-changing solution $u_{2}$ that satisfies

$$
\int_{\Omega}\left(\lambda\left|u_{2}\right|^{r-p}+\mu \frac{\left|u_{2}\right|^{q-p}}{|x|^{s}}\right) v\left(u_{2}\right)^{p-1} u_{2}=0,
$$

where $v\left(u_{2}\right)$ is the first eigenvector of the (weighted) eigenvalue problem

$$
\left\{\begin{aligned}
-\triangle_{p} v & =\nu\left(\lambda\left|u_{2}\right|^{r-p}+\mu \frac{\left|u_{2}\right|^{q-p}}{|x|^{s}}\right)|v|^{p-2} v \quad \text { in } \Omega \\
v & =0 \text { on } \partial \Omega
\end{aligned}\right.
$$

Proof. Now that under these conditions the functional $E_{\lambda, \mu}$ satisfies $(\mathrm{PS})_{c}$ for any c. It is now enough to apply Theorem 5.1 to $\mathcal{F}_{1}$ and its dual set $M_{1}$ (resp., to $\mathcal{F}_{2}$ and its dual set $M_{2}$ ) to get a solution $u_{1}$ (resp. $u_{2}$ ) which minimizes the energy functional on $M_{1}$ (resp. $M_{2}$ ).

To obtain other solutions, we need the following result of Rabinowitz ([17]).

Lemma 6.1. Let $E$ be an even $C^{1}$-functional satisfying the Palais-Smale condition on a Banach space $X=Y \oplus Z$ with $\operatorname{dim}(Y)<\infty$. Assume $E(0)=0$, as well as the following conditions:

(1) There is $\rho>0$ such that $\inf _{S_{\rho}(Z)} E \geq 0$.

(2) There exists an increasing sequence $\left\{Y_{n}\right\}_{n}$ of finite dimensional subspaces of $X$, all containing $Y$, such that $\lim _{n} \operatorname{dim}\left(Y_{n}\right)=\infty$ and for each $n, \sup _{S_{R_{n}}\left(Y_{n}\right)} E$ $\leq 0$ for some $R_{n}>\rho$.

Then $E$ has an unbounded sequence of critical values.

We now show that the functional

$$
E(u)=\frac{1}{p} \int_{\Omega}|\nabla u|^{p}-\frac{\mu}{q} \int_{\Omega} \frac{|u|^{q}}{|x|^{s}}-\frac{\lambda}{r} \int_{\Omega}|u|^{r}
$$

satisfies the hypothesis of the lemma.

Without loss of generality, we assume that $\Omega=(0,1)^{n}$. Let $Y_{k}$ be the $k$ dimensional subspace of $X=H_{0}^{1, p}(\Omega)$, generated by the first $k$ functions of the basis

$$
\left\{\left(\sin k_{1} \pi x_{1}, \cdots, \sin k_{n} \pi x_{n}\right), k_{i} \in \mathbf{N}, i=1, \cdots, n\right\} .
$$

Let $Z_{k}$ denote the complement of $Y_{k}$ in $X$, that is, the set generated by the base vectors not in $Y_{k}$. For any $u \in Y_{k-1}^{c}$, the topological complement of $Y_{k-1}$,

$$
\|u\|_{p} \leq C\|\nabla u\|_{p} / k^{\frac{1}{n}} \quad(\text { Peral [25]). }
$$

Claim 1. For $k$ sufficiently large, there exists $\rho>0$ such that $E(u) \geq 1$ for all $u \in Z_{k-1}$ with $\|u\|_{H_{0}^{1, p}}=\rho$

Proof of Claim 1. We first consider the case where $p=q, p<r$ and $\mu<\mu_{s, p}$ :

$$
E(u) \geq\left(1-\frac{\mu}{\mu_{s, p}}\right) \frac{1}{p} \int_{\Omega}|\nabla u|^{p}-C \int_{\Omega}|u|^{r} .
$$

By the Gagliardo-Nirenberg inequality,

$$
\left(\int_{\Omega}|u|^{r}\right)^{\frac{1}{r}} \leq C_{1}\left(\int_{\Omega}|\nabla u|^{p}\right)^{\frac{a}{p}}\left(\int_{\Omega}|u|^{p}\right)^{\frac{1-a}{p}}
$$


with $a=\frac{n}{p}\left(1-\frac{p}{r}\right)$. Hence, for $u \in \partial B_{\rho} \cap Y_{k-1}^{c}$,

$$
\begin{aligned}
E(u) & \geq C_{2} \int_{\Omega}|\nabla u|^{p}-C_{1}\left(\int_{\Omega}|\nabla u|^{p}\right)^{\frac{r a}{p}}\left(\int_{\Omega}|u|^{p}\right)^{\frac{r(1-a)}{p}} \\
& =\rho^{p}\left(C_{2}-C_{1} \rho^{r a}\left(\frac{C \rho}{k^{\frac{1}{n}}}\right)^{r(1-a)} \rho^{-p}\right) \\
& =\rho^{p}\left(C_{2}-C_{3} \rho^{r-p} \frac{1}{k^{r(1-a) / n}}\right) .
\end{aligned}
$$

Choosing $\rho=\left(\frac{C_{2}}{2 C_{3}} k^{\frac{r(1-a)}{n}}\right)^{\frac{1}{r-p}}$, we get that $E(u) \geq \frac{1}{2} C_{2} \rho^{p}=C_{4} k^{\frac{p r(1-a)}{n(r-p)}} \geq 1$, for $k$ large enough. This completes the proof of Claim 1 in the first case.

We turn to the case where $p<q$ : Since $q<p^{*}(s)$, choose $\varepsilon>0$ such that $q<\frac{n-s-\varepsilon}{n-p} p ;$ then

$$
\int_{\Omega} \frac{|u|^{q}}{|x|^{s}} \leq C_{0}\left(\int_{\Omega}|u|^{q \frac{n}{n-s-\varepsilon}}\right)^{\frac{n-s-\varepsilon}{n}} .
$$

If $q \frac{n}{n-s-\varepsilon} \leq r$, then

$$
\int_{\Omega}|u|^{q \frac{n}{n-s-\varepsilon}} \leq C_{1}\left(\int_{\Omega}|u|^{r}\right)^{\frac{q \frac{n}{n-s-\varepsilon}}{r}} .
$$

Thus

$$
\int_{\Omega} \frac{|u|^{q}}{|x|^{s}} \leq C_{2}\left(\int_{\Omega}|u|^{r}\right)^{\frac{q}{r}}
$$

If $q \frac{n}{n-s-\varepsilon} \geq r$, then

$$
\int_{\Omega}|u|^{r} \leq C_{3}\left(\int \Omega|u|^{q \frac{n}{n-s-\varepsilon}}\right)^{\frac{n-s-\varepsilon}{n} \cdot \frac{r}{q}} .
$$

Because of these relationships, we could combine the last two terms of the functional $E$ together. In this sense, we may assume that

$$
E(u) \geq \frac{1}{p} \int_{\Omega}|\nabla u|^{p}-C \int_{\Omega}|u|^{r},
$$

and the rest is as in case (1).

Let $Y=Y_{k}$ with the $k$ chosen in Claim 1. We now show the following.

Claim 2. In both cases, there exist for each finite dimensional subspace $Y_{k} \subset$ $H_{0}^{1, p}(\Omega)$, positive constants $C_{1}, C_{2}$ (depending on $Y_{k}$ ) such that

$$
\sup _{u \in \partial B_{R}\left(Y_{k}\right)} E(u) \leq C_{1} R^{p}-C_{2} R^{r} .
$$

Indeed, for any $u \in H_{0}^{1, p}(\Omega)$ and any $R>0$, we have

$$
E(R u) \leq \frac{R^{p}}{p}\|u\|_{H_{0}^{1, p}(\Omega)}^{p}-\frac{R^{r}}{r}\|u\|_{r}^{r} .
$$

Since $Y_{k}$ is a finite dimensional space, it is closed and the two norms $\|\cdot\|_{r}$ and $\|\cdot\|_{H_{0}^{1, p}(\Omega)}$ on $Y_{k}$ are equivalent. This implies the Claim.

Now we can apply Lemma 6.1 to conclude that $E_{\lambda, \mu}$ has an unbounded sequence of critical values. Theorem 6.1 is proved. 


\section{The SOLUTIONS IN THE CASE OF}

A HARDY-CRITICAL SINGULAR TERM

Theorem 7.1 (Hardy-critical singular term). Suppose $1<p=q=p^{*}(s)$ (i.e., $s=p)$.

(1) If $p<r<p^{*}$ (high order non-singular term), then $\left(\mathrm{P}_{\lambda, \mu}\right)$ has infinitely many solutions - at least one of them being positive- for any $\lambda>0$ and any $0<\mu<\mu_{p}$.

(2) If $r=p^{*}$ (critical non-singular term) and $\Omega$ is star-shaped, then $\left(\mathrm{P}_{\lambda, \mu}\right)$ has no non-trivial solution for any $\lambda>0, \mu>0$.

Proof. If $r<p^{*}$, then by Theorem 4.1.2 the functional $E_{\lambda, \mu}$ satisfies (PS) $)_{c}$ for any $c$ as long as $\mu<\mu_{p}$. Since $p<r$, the proof is the same as in Theorem 6.1.(2), while the second case of the theorem is covered in section 2 .

Remark 7.1. The case when $p=q=r$ is really an eigenvalue problem. There are solutions for $\left(P_{\lambda, \mu}\right)$ as long as $\lambda$ is an eigenvalue of the problem $-\Delta_{p} u-\frac{\mu|u|^{p-2} u}{|x|^{p}}=$ $\lambda|u|^{p-2} u$ in $H_{0}^{1, p}(\Omega)$.

If $0 \leq \mu<\mu_{p}$, one can show that there is an infinite number of eigenvalues for the above problem. Indeed, these correspond to the critical levels of the restriction of the functional

$$
\tilde{E}(u)=\frac{1}{p} \int_{\Omega}|\nabla u|^{p} d x-\frac{\mu}{p} \int_{\Omega} \frac{|u|^{p}}{|x|^{p}} d x
$$

to the submanifold $\left\{u ; \int_{\Omega}|u|^{p} d x=1\right\}$. But a slight variation of Theorem 4.1.(2) shows that in this case $\widetilde{E}$ has $(\mathrm{PS})_{c}$ for any $c$, and therefore a standard application of Ljusternik-Schnirelmann theory applied to the genus $\gamma_{\mathbf{z}_{2}}$ will yield the result.

\section{A positive SOlution in the Case of A HARDY-Sobolev CRITICAL SINGUlar TERM}

In this section, we consider the first solution for the problem $\left(\mathrm{P}_{\lambda, \mu}\right)$ with the critical Sobolev-Hardy exponent.

Theorem 8.1 (Hardy-Sobolev critical singular term). Suppose $1<p<q=p^{*}(s)$ (i.e., $s<p$ ) in the equation:

$\left(\mathrm{P}_{\lambda, \mu}\right) \quad\left\{\begin{aligned}-\triangle_{p} u & =\lambda|u|^{r-2} u+\mu \frac{|u|^{p^{*}(s)-2}}{|x|^{s}} u \quad \text { in } \Omega, \\ \left.u\right|_{\partial \Omega} & =0 .\end{aligned}\right.$

- If $r<p^{*}$, then $\left(\mathrm{P}_{\lambda, \mu}\right)$ has a solution that is strictly positive everywhere on $\Omega$, under any one of the following conditions:

(1) $p=r<p^{*}$ and $n \geq p^{2}, 0<\lambda<\lambda_{1}$ and $\mu>0$.

(2) $p<r<p^{*}, \lambda$ is large enough and $\mu>0$.

(3) $p<r<p^{*}$ and $n>\frac{p(p-1) r+p^{2}}{p+(p-1)(r-p)}, \lambda>0, \mu>0$.

- If $r=p^{*}$ and $\Omega$ is star-shaped, then $\left(\mathrm{P}_{\lambda, \mu}\right)$ has no non-trivial solution for any $\lambda>0, \mu>0$.

Proof. Note that the last case $\left(r=p^{*}\right)$ was covered in section 2. Now if $r<p^{*}$, then Theorem 5.2 asserts that any one of the 3 conditions yields that the set

$$
M_{1}=\left\{u \in H_{0}^{1, p}(\Omega) ; u \neq 0,\left\langle E_{\lambda, \mu}^{\prime}(u), u\right\rangle=0\right\},
$$


is closed, that it is dual to the Mountain Pass class

$$
\mathcal{F}_{1}=\left\{\gamma \in C\left([0,1] ; H_{0}^{1, p}(\Omega)\right) ; \gamma(0)=0, \gamma(1) \neq 0 \text { and } E_{\lambda, \mu}(\gamma(1)) \leq 0\right\},
$$

and that

$$
\inf _{M_{1}} E_{\lambda, \mu}=c_{1}:=c\left(E_{\lambda, \mu}, \mathcal{F}_{1}\right) .
$$

On the other hand, Theorem 4.1.(2) yields that $E_{\lambda, \mu}$ satisfies the (PS) condition for any

$$
c<\frac{p-s}{p(n-s)} \mu_{s}^{\frac{n-s}{p-s}} .
$$

Therefore, we should be able to apply Theorem 5.1 and obtain our desired assertion, if only we can prove the following case.

Lemma 8.1. In any one of the above three cases, we have

$$
c_{1}<\frac{p-s}{p(n-s)}\left(\frac{\mu_{s}^{n-s}}{\mu^{n-p}}\right)^{\frac{1}{p-s}} .
$$

Proof. We may assume without loss of generality that $\mu=1$. We first consider the following case:

Case (1). $p<r$ and $\lambda$ is large.

In order to estimate the energy level $c_{1}$, we consider the functions

$$
g(t)=E_{\lambda, \mu}\left(t v_{\varepsilon}\right)=\frac{t^{p}}{p} \int_{\Omega}\left|\nabla v_{\varepsilon}\right|^{p}-\frac{t^{p^{*}(s)}}{p^{*(s)}}-\frac{\lambda t^{r}}{r} \int_{\Omega}\left|v_{\varepsilon}\right|^{r}
$$

and

$$
\bar{g}(t)=\frac{t^{p}}{p} \int_{\Omega}\left|\nabla v_{\varepsilon}\right|^{p}-\frac{t^{p^{*}(s)}}{p^{*}(s)}
$$

where $v_{\varepsilon}$ is the extremal function defined in the appendix. Note that $\lim _{t \rightarrow \infty} g(t)=$ $-\infty$ and $g(t)>0$ when $t$ is close to 0 , so that $\sup _{t \geq 0} g(t)$ is attained for some $t_{\varepsilon}>0$. From

we have

$$
0=g^{\prime}\left(t_{\varepsilon}\right)=t_{\varepsilon}^{p-1}\left(\int_{\Omega}\left|\nabla v_{\varepsilon}\right|^{p}-t_{\varepsilon}^{p^{*}(s)-p}-\lambda t_{\varepsilon}^{r-p} \int_{\Omega}\left|v_{\varepsilon}\right|^{r}\right)
$$

and therefore

$$
\int_{\Omega}\left|\nabla v_{\varepsilon}\right|^{p}=t_{\varepsilon}^{p^{*}(s)-p}+\lambda t_{\varepsilon}^{r-p} \int_{\Omega}\left|v_{\varepsilon}\right|^{r}>t_{\varepsilon}^{p^{*}(s)-p}
$$

Thus

$$
t_{\varepsilon} \leq\left(\int_{\Omega}\left|\nabla v_{\varepsilon}\right|^{p}\right)^{\frac{1}{p^{*}(s)-p}}
$$

$$
\int_{\Omega}\left|\nabla v_{\varepsilon}\right|^{p} \leq t_{\varepsilon}^{p^{*}(s)-p}+\lambda\left(\int_{\Omega}\left|\nabla v_{\varepsilon}\right|^{p}\right)^{\frac{r-p}{p^{*}(s)-p}}\left(\int_{\Omega}\left|v_{\varepsilon}\right|^{r}\right) .
$$

Choose $\varepsilon$ small enough so that by (1) and (6) of Lemma 11.1 we have $t_{\varepsilon}^{p^{*}(s)-p} \geq \frac{\mu_{s}}{2}$. That is, we get a lower bound for $t_{\varepsilon}$, which is independent of $\varepsilon$.

Now we estimate $g\left(t_{\varepsilon}\right)$. The function $\bar{g}(t)$ attains its maximum at

$$
t=\left(\int_{\Omega}\left|\nabla v_{\varepsilon}\right|^{p}\right)^{\frac{1}{p^{*}(s)-p}}
$$

and is increasing in the interval

$$
\left[0, \quad\left(\int_{\Omega}\left|\nabla v_{\varepsilon}\right|^{p}\right)^{\frac{1}{p^{*}(s)-p}}\right] .
$$


By Lemma 11.1, we have

$$
\begin{aligned}
g\left(t_{\varepsilon}\right) & =\bar{g}\left(t_{\varepsilon}\right)-\frac{\lambda}{r} t_{\varepsilon}^{r} \int_{\Omega}\left|v_{\varepsilon}\right|^{r} \\
& \leq \bar{g}\left(\left(\int_{\Omega}\left|\nabla v_{\varepsilon}\right|^{p}\right)^{\frac{1}{p^{*}(s)-p}}\right)-\frac{\lambda}{r} t_{\varepsilon}^{r} \int_{\Omega}\left|v_{\varepsilon}\right|^{r} \\
& =\left(\frac{1}{p}-\frac{1}{p^{*}(s)}\right)\left(\int_{\Omega}\left|\nabla v_{\varepsilon}\right|^{p}\right)^{\frac{p^{*}(s)}{p^{*}(s)-p}}-\frac{\lambda}{r} t_{\varepsilon}^{r} \int_{\Omega}\left|v_{\varepsilon}\right|^{r} \\
& \leq \frac{p-s}{p(n-s)} \mu_{s}^{\frac{n-s}{p-s}}+O\left(\varepsilon^{\frac{n-p}{p-s}}\right)-\frac{\lambda}{r}\left(\frac{\mu_{s}}{2}\right)^{\frac{r}{p^{*}(s)-p}} \int_{\Omega}\left|v_{\varepsilon}\right|^{r} .
\end{aligned}
$$

So for $\lambda$ large enough, we have

$$
g\left(t_{\varepsilon}\right)<\frac{p-s}{p(n-s)} \mu_{s}^{\frac{n-s}{p-s}}
$$

Case (2). $p<r<p^{*}$ and $n>\frac{p(p-1) r+p^{2}}{p+(p-1)(r-p)}, \lambda>0$.

Note first that the above condition is equivalent to $\max \left\{p, p^{*}-\frac{p}{p-1}\right\}<r<p^{*}$ and $\lambda>0$.

For any $\lambda>0$, the above estimate on $g\left(t_{\varepsilon}\right)$ and Lemma 6.1 yield

$$
g\left(t_{\varepsilon}\right) \leq \frac{p-s}{p(n-s)} \mu_{s}^{\frac{n-s}{p-s}}+O\left(\varepsilon^{\frac{n-p}{p-s}}\right)-O\left(\varepsilon^{\frac{p-1}{p-s}\left(n-\frac{r(n-p)}{p}\right)}\right),
$$

so that if $r$ is chosen in such a way that

$$
\frac{n-p}{p-s}>\frac{p-1}{p-s}\left(n-\frac{r(n-p)}{p}\right)
$$

i.e. $r>p^{*}-\frac{p}{p-1}$, then

$$
g\left(t_{\varepsilon}\right)<\frac{p-s}{p(n-s)} \mu_{s}^{\frac{n-s}{p-s}}
$$

Case (3). $p=r, 0<\lambda<\lambda_{1}$ and $n \geq p^{2}$.

We still use the function $g(t)$. Since $\lambda<\lambda_{1}$, we have $g(t)>0$ when $t$ is close to 0 , and $\lim _{t \rightarrow \infty} g(t)=-\infty$. So again $g(t)$ attains its maximum at some $t_{\varepsilon}>0$. From

$$
g^{\prime}(t)=t^{p-1}\left(\int_{\Omega}\left|\nabla v_{\varepsilon}\right|^{p}-t^{p^{*}(s)-p}-\lambda \int_{\Omega}\left|v_{\varepsilon}\right|^{p}\right)=0
$$

we get

$$
t_{\varepsilon}=\left(\int_{\Omega}\left|\nabla v_{\varepsilon}\right|^{p}-\lambda \int_{\Omega}\left|v_{\varepsilon}\right|^{p}\right)^{\frac{1}{p^{*}(s)-p}}
$$

Thus

$$
\begin{aligned}
g\left(t_{\varepsilon}\right) & =\left(\frac{1}{p}-\frac{1}{p^{*}(s)}\right)\left(\int_{\Omega}\left|\nabla v_{\varepsilon}\right|^{p}-\lambda \int_{\Omega}\left|v_{\varepsilon}\right|^{p}\right)^{\frac{p^{*}(s)}{p^{*}(s)-p}} \\
& = \begin{cases}\frac{p-s}{p(n-s)} \mu_{s}^{\frac{n-s}{p-s}}+O\left(\varepsilon^{\frac{n-p}{p-s}}\right)-O\left(\varepsilon^{\frac{p(p-1)}{p-s}}\right), & p>p^{*}\left(1-\frac{1}{p}\right), \\
\frac{p-s}{p(n-s)} \mu_{s}^{\frac{n-s}{p-s}}+O\left(\varepsilon^{\frac{n-p}{p-s}}\right)-O\left(\varepsilon^{\frac{n-p}{p-s}}|\log \varepsilon|\right), & p=p^{*}\left(1-\frac{1}{p}\right) .\end{cases}
\end{aligned}
$$


In the case where $p>p^{*}\left(1-\frac{1}{p}\right)$, we require that $\frac{n-p}{p-s}>\frac{p(p-1)}{p-s}$, but both are equivalent to $p^{2}<n$. In the case where $p=p^{*}\left(1-\frac{1}{p}\right)$ we have $p^{2}=n$, and the proof the lemma is now complete.

Remark 8.1. (1) If $p^{2} \leq n$, then $p^{*}-\frac{p}{p-1} \leq p^{*}\left(1-\frac{1}{p}\right) \leq p$, so that $r>p^{*}-\frac{p}{p-1}$ whenever $p<r$. In this case, $r$ can take any value between $p$ and $p^{*}$.

(2) If $p^{2}>n$, then $p<p^{*}\left(1-\frac{1}{p}\right)<p^{*}-\frac{p}{p-1}$, and then we require that $p^{*}-\frac{p}{p-1}<$ $p<p^{*}$.

\section{A Sign Changing SOlution in the Hardy-Sobolev CRitical Case}

In this section, we extend the arguments of Tarantello [28] to establish the following.

Theorem 9.1 (Hardy-Sobolev critical singular term). Suppose $2 \leq p<q=p^{*}(s)$ and $r<p^{*}$ in the equation

$\left(\mathrm{P}_{\lambda, \mu}\right)$

$$
\left\{\begin{aligned}
-\triangle_{p} u & =\lambda|u|^{r-2} u+\mu \frac{|u|^{p^{*}(s)-2}}{|x|^{s}} u \text { in } \Omega, \\
\left.u\right|_{\partial \Omega} & =0 .
\end{aligned}\right.
$$

Assume any one of the following conditions:

(1) $p=r<p^{*}, n>p^{3}-p^{2}+p, \mu>0$ and $0<\lambda<\lambda_{1}$.

(2) $p<r<p^{*}, \mu>0$ and $\lambda$ large enough.

(3) $p<r<p^{*}, n>\frac{p(p-1) r+p}{1+(p-1)(r-p)}$ and $\mu>0, \lambda>0$.

Then $\left(\mathrm{P}_{\lambda, \mu}\right)$ has also a changing-sign solution $u$ that satisfies

$$
\int_{\Omega}\left(\lambda|u|^{r-p}+\mu \frac{|u|^{p^{*}(s)-p}}{|x|^{s}}\right) v(u)^{p-1} u=0,
$$

where $v(u)$ is the first eigenvector of the (weighted) eigenvalue problem

$$
\left\{\begin{aligned}
-\triangle_{p} v & =\nu\left(\lambda|u|^{r-p}+\mu \frac{|u|^{p^{*}(s)-p}}{|x|^{s}}\right)|v|^{p-2} v \quad \text { in } \Omega \\
v & =0 \text { on } \partial \Omega .
\end{aligned}\right.
$$

Proof. We assume without loss of generality that $\mu=1$. Theorem 5.2 asserts that for any $q<p^{*}(s)$, any one of the 3 conditions yields that the closed set

$$
M_{2}^{q}=M_{1}^{q} \cap\left\{u \in H_{0}^{1, p}(\Omega) ; \int_{\Omega}\left(\lambda|u|^{r-p}+\frac{|u|^{q-p}}{|x|^{s}}\right) v(u)^{p-1} u=0\right\} .
$$

is dual to the class $\mathcal{F}_{2}$, and that

$$
\inf _{M_{2}^{q}} E_{\lambda, q}=c_{2, q}:=c\left(E_{\lambda, q}, \mathcal{F}_{2}\right),
$$

where for each $q<q^{*}(s)$, the sets $M_{1}^{q}$ (resp. $M_{2}^{q}$ ) denote the dual sets associated to the functional

$$
E_{\lambda, q}:=\frac{1}{p} \int_{\Omega}|\nabla u|^{p}-\frac{1}{q} \int_{\Omega} \frac{|u|^{q}}{|x|^{s}}-\frac{\lambda}{r} \int_{\Omega}|u|^{r} .
$$

$E_{\lambda}$ will denote $E_{\lambda, p^{*}(s)}$ and $M_{1}\left(\operatorname{resp} . M_{2}\right)$ will denote $M_{1}^{p^{*}(s)}$ (resp. $M_{2}^{p^{*}(s)}$ ). Note that by Theorem 5.2, $M_{1}$ is dual to $\mathcal{F}_{1}$, but the same cannot be said about $M_{2}$ and $\mathcal{F}_{2}$ unless $p=2$. Therefore, to establish the theorem above, we shall resort to a limiting argument as $q \rightarrow p^{*}(s)$. 
Lemma 9.1. Under any one of the 3 conditions in Theorem 9.1, we have:

(1) $c_{i, q} \rightarrow c_{i}(i=1,2)$ as $q \rightarrow p^{*}(s)$.

(2) There exist $\sigma>0$ and $\delta_{0}>0$ such that for $0<\left|q-p^{*}(s)\right|<\delta_{0}$, we have $c_{2, q} \leq c_{1, q}+\frac{1}{n} S^{\frac{n}{p}}-\sigma$.

Proof. (1) First we prove that $\left(c_{1, q}\right)_{q}$ and $\left(c_{2, q}\right)_{q}$ are uniformly bounded in $q$. We shall only show it for $c_{2, q}$. For any $u \in M_{2}^{q}$,

$$
\int_{\Omega}|\nabla u|^{p}-\int_{\Omega} \frac{|u|^{q}}{|x|^{s}}-\lambda \int_{\Omega}|u|^{r}=0 .
$$

Thus

$$
E_{\lambda, q}(u)=\lambda\left(\frac{1}{p}-\frac{1}{r}\right) \int_{\Omega}|u|^{r}+\left(\frac{1}{p}-\frac{1}{q}\right) \int_{\Omega} \frac{|u|^{q}}{|x|^{s}} d x \geq 0,
$$

i.e., $c_{2, q} \geq \inf _{M_{2}^{q}} E_{\lambda, q} \geq 0$. Since now

$$
\begin{aligned}
E_{\lambda, q}(u) & =\frac{1}{p} \int_{\Omega}|\nabla u|^{p}-\frac{1}{q} \int_{\Omega} \frac{|u|^{q}}{|x|^{s}}-\frac{\lambda}{r} \int_{\Omega}|u|^{r} \\
& \leq \frac{1}{p} \int_{\Omega}|\nabla u|^{p}-\frac{\lambda}{r} \int_{\Omega}|u|^{r} \equiv E(u),
\end{aligned}
$$

and for any $u_{0}, v_{0} \in H_{0}^{1, p}(\Omega)$,

$$
\begin{aligned}
& \lim _{\alpha \rightarrow \infty, \beta \rightarrow \infty} E\left(\alpha u_{0}+\beta v_{0}\right) \\
& \quad=\lim _{\alpha \rightarrow \infty, \beta \rightarrow \infty}\left(\frac{1}{p} \int_{\Omega}\left|\nabla\left(\alpha u_{0}+\beta v_{0}\right)\right|^{p}-\frac{\lambda}{r} \int_{\Omega}\left|\alpha u_{0}+\beta v_{0}\right|^{r}\right)=-\infty,
\end{aligned}
$$

$E\left(\alpha u_{0}+\beta v_{0}\right)$ attains its maximum at some finite $\alpha_{0}$ and $\beta_{0}$. This means

$$
0 \leq c_{2, q} \leq E\left(\alpha_{0} u_{0}+\beta_{0} v_{0}\right)
$$

which is independent of $q$.

This implies the existence of constants $C_{1}>0$ and $C_{2}>0$ such that

$$
C_{1} \leq \int_{\Omega} \frac{\left|u_{1, q}\right|^{q}}{|x|^{s}} \leq C_{2}
$$

Similar estimates also hold for $\left\|\nabla u_{1, q}\right\|_{p}$ and $\left\|u_{1, q}\right\|_{r}$. Notice that for every $u \neq 0$, there exist unique $t_{q}(u)>0$ and $t(u)>0$ such that

$$
t(u) u \in M_{1} \quad \text { and } \quad t_{q}(u) u \in M_{1}^{q}
$$

Furthermore, $t_{q}(u) \rightarrow t(u)$ as $q \rightarrow p^{*}(s)$. Set $s_{q}=t\left(u_{1, q}\right)$ so that $s_{q} u_{1, q} \in M_{1}$. We have

$$
\begin{aligned}
c_{1} & \leq E_{\lambda}\left(s_{q} u_{1, q}\right) \\
& =\frac{1}{p} \int_{\Omega}\left|\nabla s_{q} u_{1, q}\right|^{p}-\frac{\lambda}{r} \int_{\Omega}\left|s_{q} u_{1, q}\right|^{r}-\frac{1}{p^{*}(s)} \int_{\Omega} \frac{\left|s_{q} u_{1, q}\right|^{p^{*}(s)}}{|x|^{s}} \\
& =\left(\frac{1}{p}-\frac{1}{p^{*}(s)}\right) \int_{\Omega}\left|\nabla s_{q} u_{1, q}\right|^{p}+\lambda\left(\frac{1}{p^{*}(s)}-\frac{1}{r}\right) \int_{\Omega}\left|s_{q} u_{1, q}\right|^{r} .
\end{aligned}
$$


Since $u_{1, q} \in M_{1}^{q}$, we have

$$
\begin{aligned}
E_{\lambda, q}\left(u_{1, q}\right) & =\frac{1}{p} \int_{\Omega}\left|\nabla u_{1, q}\right|^{p}-\frac{\lambda}{r} \int_{\Omega}\left|u_{1, q}\right|^{r}-\frac{1}{q} \int_{\Omega} \frac{\left|u_{1, q}\right|^{q}}{|x|^{s}} \\
& =\left(\frac{1}{p}-\frac{1}{q}\right) \int_{\Omega}\left|\nabla u_{1, q}\right|^{p}+\lambda\left(\frac{1}{q}-\frac{1}{r}\right) \int_{\Omega}\left|u_{1, q}\right|^{r} .
\end{aligned}
$$

Thus

$$
\begin{aligned}
c_{1} \leq & E_{\lambda}\left(s_{q} u_{1, q}\right) \\
= & \left(\frac{1}{p}-\frac{1}{q}\right) \int_{\Omega} s_{q}^{p}\left|\nabla u_{1, q}\right|^{p}+\left(\frac{1}{q}-\frac{1}{p^{*}(s)}\right) \int_{\Omega} s_{q}^{p}\left|\nabla u_{1, q}\right|^{p} \\
& +\lambda\left(\frac{1}{p^{*}(s)}-\frac{1}{r}\right) \int_{\Omega}\left|s_{q} u_{1, q}\right|^{r} \\
= & \left(\frac{1}{p}-\frac{1}{q}\right) \int_{\Omega}\left|\nabla u_{1, q}\right|^{p}+\left(\frac{1}{p}-\frac{1}{q}\right)\left(s_{q}^{p}-1\right) \int_{\Omega}\left|\nabla u_{1, q}\right|^{p} \\
& +\left(\frac{1}{q}-\frac{1}{p^{*}(s)}\right) \int_{\Omega} s_{q}^{p}\left|\nabla u_{1, q}\right|^{p}+\lambda\left(\frac{1}{p^{*}(s)}-\frac{1}{r}\right) \int_{\Omega}\left|s_{q} u_{1, q}\right|^{r} \\
= & E_{\lambda, q}\left(u_{1, q}\right)+\left(\frac{1}{p}-\frac{1}{q}\right)\left(s_{q}^{p}-1\right) \int_{\Omega}\left|\nabla u_{1, q}\right|^{p} \\
& +\left(\frac{1}{q}-\frac{1}{p^{*}(s)}\right) \int_{\Omega} s_{q}^{p}\left|\nabla u_{1, q}\right|^{p} \\
& \lambda\left(\frac{1}{q}-\frac{1}{r}\right)\left(s_{q}^{r}-1\right) \int_{\Omega}\left|u_{1, q}\right|^{r}+\lambda\left(\frac{1}{p^{*}(s)}-\frac{1}{q}\right) \int_{\Omega} s_{q}^{r}\left|u_{1, q}\right|^{r} .
\end{aligned}
$$

Note that $s_{q} \rightarrow 1$ as $q \rightarrow p^{*}(s)$; therefore

$$
c_{1} \leq c_{1, q}+o(1)
$$

To obtain the reverse inequality, set $t_{q}=t_{q}\left(u_{1}\right)>0$. Thus, $t_{q} u_{1} \in M_{1}^{q}, t_{q} \rightarrow 1$ as $q \rightarrow p^{*}(s)$, and

$$
\begin{aligned}
c_{1, q} \leq E_{\lambda, q}\left(t_{q} u_{1}\right) & =E_{\lambda}\left(t_{q} u_{1}\right)+\frac{1}{p^{*}(s)} \int_{\Omega} \frac{|u|^{p^{*}(s)}}{|x|^{s}}-\frac{1}{q} \int_{\Omega} \frac{|u|^{q}}{|x|^{s}} \\
& =E_{\lambda}\left(u_{1}\right)+o(1)=c_{1}+o(1) .
\end{aligned}
$$

This completes part (1) of the lemma.

We prove part (2) by estimating $\sup _{A_{\varepsilon}} E_{\lambda, q}$, where $A_{\varepsilon}=\operatorname{span}\left\{u_{1}, v_{\varepsilon}\right\} \in \mathcal{F}_{2}$ and $u_{1}$ is the first solution of the critical problem. For that, we need the smoothness of $u_{1}$; but this cannot be guaranteed unless $p=2$. However, an easy approximation argument, and the fact that $\sup _{t \geq 0} E_{\lambda}(t u) \rightarrow \sup _{t \geq 0} E\left(t u_{1}\right)$ as $u \rightarrow u_{1}$ strongly, allow us to assume that $u_{1}$ has the required smoothness.

Therefore we may suppose that $u_{1}, \nabla u_{1} \in L^{\infty}(\Omega)$. We shall consider the case where $r>p$ first.

Case (1). Assume $p<r<p^{*}$ and $\lambda>0$. 
For $\varepsilon>0$ and $q$ sufficiently close to $p^{*}(s)$, and by the calculus lemma,

$$
\begin{aligned}
E_{\lambda, q}(\alpha & \left.u_{1}+\beta v_{\varepsilon}\right) \\
= & \frac{1}{p} \int_{\Omega}\left|\nabla\left(\alpha u_{1}+\beta v_{\varepsilon}\right)\right|^{p}-\frac{\lambda}{r} \int_{\Omega}\left|\alpha u_{1}+\beta v_{\varepsilon}\right|^{r}-\frac{1}{q} \int_{\Omega} \frac{\left|\alpha u_{1}+\beta v_{\varepsilon}\right|^{q}}{|x|^{s}} \\
\leq & \frac{1}{p} \int_{\Omega}\left|\nabla\left(\alpha u_{1}\right)\right|^{p}-\frac{\lambda}{r} \int_{\Omega}\left|\alpha u_{1}\right|^{r}-\frac{1}{q} \int_{\Omega} \frac{\left|\alpha u_{1}\right|^{q}}{|x|^{s}} \\
& +\frac{1}{p} \int_{\Omega}\left|\nabla\left(\beta v_{\varepsilon}\right)\right|^{p}-\frac{\lambda}{r} \int_{\Omega}\left|\beta v_{\varepsilon}\right|^{r}-\frac{1}{q} \int_{\Omega} \frac{\left|\beta v_{\varepsilon}\right|^{q}}{|x|^{s}} \\
& +A_{1}\left[\int_{\Omega}\left|\nabla \alpha u_{1}\right|\left|\nabla \beta v_{\varepsilon}\right|^{p-1}+\left|\nabla \alpha u_{1}\right|^{p-1}\left|\nabla \beta v_{\varepsilon}\right|\right] \\
& +B_{1}\left[\int_{\Omega}\left|\alpha u_{1}\right|\left|\beta v_{\varepsilon}\right|^{r-1}+\left|\alpha u_{1}\right|^{r-1}\left|\beta v_{\varepsilon}\right|\right] \\
& +C_{1}\left[\int_{\Omega}\left|\alpha u_{1}\right| \frac{\left|\beta v_{\varepsilon}\right|^{q-1}}{|x|^{s}}+\left|\alpha u_{1}\right|^{q-1} \frac{\left|\beta v_{\varepsilon}\right|}{\left.|x|^{s}\right]}\right. \\
\leq & \frac{1}{p} \int_{\Omega}\left|\nabla\left(\alpha u_{1}\right)\right|^{p}-\frac{\lambda}{r} \int_{\Omega}\left|\alpha u_{1}\right|^{r}-\frac{1}{q} \int_{\Omega} \frac{\left|\alpha u_{1}\right|^{q}}{|x|^{s}} \\
& +\frac{1}{p} \int_{\Omega}\left|\nabla\left(\beta v_{\varepsilon}\right)\right|^{p}-\frac{\lambda}{r} \int_{\Omega}\left|\beta v_{\varepsilon}\right|^{r}-\frac{1}{q} \int_{\Omega} \frac{\left|\beta v_{\varepsilon}\right|^{q}}{|x|^{s}} \\
& +A_{2}\left(|\alpha|^{p}+|\beta|^{p}\right) \varepsilon^{\frac{n-p}{p(p-s)}}+B_{2}\left(|\alpha|^{r}+|\beta|^{r}\right) \varepsilon^{\frac{p-1}{p-s}\left(n-\frac{(r-1)(n-p)}{p}\right)} \\
& +C_{2}\left(|\alpha|^{q}+|\beta|^{q}\right) \varepsilon^{\frac{n-p}{p(p-s)}} .
\end{aligned}
$$

Therefore, for $\varepsilon$ sufficiently small,

$$
\lim _{\alpha, \beta \rightarrow \infty} E_{\lambda, q}\left(\alpha u_{1}+\beta v_{\varepsilon}\right)=-\infty
$$

So we may assume that $\alpha$ and $\beta$ are in a bounded set.

As in the study of the first solution, let us consider the function

$$
g(t)=E_{\lambda}\left(t v_{\varepsilon}\right)=\frac{t^{p}}{p} \int_{\Omega}\left|\nabla v_{\varepsilon}\right|^{p}-\frac{t^{p^{*}(s)}}{p^{*}(s)}-\frac{\lambda t^{r}}{r} \int_{\Omega}\left|v_{\varepsilon}\right|^{r}
$$

again. As in the previous section, we have

$$
g\left(t_{\varepsilon}\right) \leq \frac{p-s}{p(n-s)} \mu_{s}^{\frac{n-s}{p-s}}+C \varepsilon^{\frac{n-p}{p-s}}-\frac{\lambda}{r}\left(\frac{\mu_{s}}{2}\right)^{\frac{r}{p^{*}(s)-p}} \int_{\Omega}\left|v_{\varepsilon}\right|^{r}
$$


If now $r>p^{*}-\frac{1}{p-1}>p^{*}-1$, we have

$$
\begin{aligned}
E_{\lambda, q}\left(\alpha u_{1}+\beta v_{\varepsilon}\right) \leq & E_{\lambda, q}\left(\alpha u_{1}\right)+E_{\lambda}\left(\beta v_{\varepsilon}\right)+\frac{|\beta|^{p^{*}(s)}}{p^{*}(s)}-\frac{|\beta|^{q}}{q} \int_{\Omega} \frac{\left|v_{\varepsilon}\right|^{q}}{|x|^{s}} \\
& +A_{3} \varepsilon^{\frac{n-p}{p(p-s)}}+B_{3} \varepsilon^{\frac{p-1}{p-s}\left(n-\frac{(r-1)(n-p)}{p}\right)} \\
\leq & c_{1, q}+\frac{p-s}{p(n-s)} \mu_{s}^{\frac{n-s}{p-s}}+C \varepsilon^{\frac{n-p}{p-s}}+\frac{|\beta|^{p^{*}(s)}}{p^{*}(s)} \\
& -\frac{|\beta|^{q}}{q} \int_{\Omega} \frac{\left|v_{\varepsilon}\right|^{q}}{|x|^{s}}-\frac{\lambda}{r}\left(\frac{\mu_{s}}{2}\right)^{\frac{r}{p^{*}(s)-p}} \int_{\Omega}\left|v_{\varepsilon}\right|^{r} \\
& +A_{3} \varepsilon^{\frac{n-p}{p(n-s)}}+B_{3} \varepsilon^{\frac{p-1}{p-s}\left(n-\frac{(r-1)(n-p)}{p}\right)} \\
\leq & c_{1, q}+\frac{p-s}{p(n-s)} \mu_{s}^{\frac{n-s}{p-s}}+C \varepsilon^{\frac{n-p}{p-s}}+A_{3} \varepsilon^{\frac{n-p}{p(p-s)}} \\
& +B_{3}{\frac{p}{p^{p-s}}\left(n-\frac{(r-1)(n-p)}{p}\right)}^{p}-C_{4} \varepsilon^{\frac{p-1}{p-s}\left(n-\frac{r(n-p)}{p}\right)} \\
& +\frac{|\beta|^{p^{*}(s)}}{p^{*}(s)}-\frac{|\beta|^{q}}{q} \int_{\Omega} \frac{\left|v_{\varepsilon}\right|^{q}}{|x|^{s}} .
\end{aligned}
$$

Choose $\varepsilon$ small enough so that

$$
C \varepsilon^{\frac{n-p}{p-s}}+A_{3} \varepsilon^{\frac{n-p}{p(p-s)}}+B_{3} \varepsilon^{\frac{p-1}{p-s}\left(n-\frac{(r-1)(n-p)}{p}\right)}-C_{4} \varepsilon^{\frac{p-1}{p-s}\left(n-\frac{r(n-p)}{p}\right)} \leq-2 \sigma
$$

for some constant $\sigma>0$. Now choose $\delta_{0}>0$ small enough so that

$$
\frac{|\beta|^{p^{*}(s)}}{p^{*}(s)}-\frac{|\beta|^{q}}{q} \int_{\Omega} \frac{\left|v_{\varepsilon}\right|^{q}}{|x|^{s}}<\sigma \text { for } 0<\left|q-p^{*}(s)\right|<\delta_{0}
$$

Thus the case when $r>p$ is established.

Case (2). $r=p, p^{3}-p^{2}+p<n$ and $0<\lambda<\lambda_{1}$.

The assumption $p^{3}-p^{2}+p<n$ implies that $p^{2}<n, p>p^{*}\left(1-\frac{1}{p}\right)$ and $p-1<p^{*}\left(1-\frac{1}{p}\right)$. We assume that $\alpha$ and $\beta$ are in a bounded set, and we estimate $E_{\lambda}\left(\alpha u_{1}+\beta v_{\varepsilon}\right)$. Again let

$$
g(t)=E_{\lambda}\left(t v_{\varepsilon}\right)=\frac{t^{p}}{p} \int_{\Omega}\left|\nabla v_{\varepsilon}\right|^{p}-\frac{t^{p^{*}(s)}}{p^{*}(s)}-\frac{\lambda t^{p}}{p} \int_{\Omega}\left|v_{\varepsilon}\right|^{p}
$$

then the maximum $g\left(t_{\varepsilon}\right)$ of $g(t)$ satisfies

$$
g\left(t_{\varepsilon}\right)=\frac{p-s}{p(n-s)} \mu_{s}^{\frac{n-s}{p-s}}+O\left(\varepsilon^{\frac{n-p}{p-s}}\right)-O\left(\varepsilon^{\frac{p(p-1)}{p-s}}\right)
$$


Thus we have

$$
\begin{aligned}
& E_{\lambda, q}\left(\alpha u_{1}+\beta v_{\varepsilon}\right) \\
& =\frac{1}{p} \int_{\Omega}\left|\nabla\left(\alpha u_{1}+\beta v_{\varepsilon}\right)\right|^{p}-\frac{\lambda}{p} \int_{\Omega}\left|\alpha u_{1}+\beta v_{\varepsilon}\right|^{p}-\frac{1}{q} \int_{\Omega} \frac{\left|\alpha u_{1}+\beta v_{\varepsilon}\right|^{q}}{|x|^{s}} \\
& \leq \frac{1}{p} \int_{\Omega}\left|\nabla\left(\alpha u_{1}\right)\right|^{p}-\frac{\lambda}{p} \int_{\Omega}\left|\alpha u_{1}\right|^{p}-\frac{1}{q} \int_{\Omega} \frac{\left|\alpha u_{1}\right|^{q}}{|x|^{s}} \\
& +\frac{1}{p} \int_{\Omega}\left|\nabla\left(\beta v_{\varepsilon}\right)\right|^{p}-\frac{\lambda}{p} \int_{\Omega}\left|\beta v_{\varepsilon}\right|^{p}-\frac{1}{q} \int_{\Omega} \frac{\left|\beta v_{\varepsilon}\right|^{q}}{|x|^{s}} \\
& +A_{1}\left[\int_{\Omega}\left|\nabla \alpha u_{1}\right|\left|\nabla \beta v_{\varepsilon}\right|^{p-1}+\left|\nabla \alpha u_{1}\right|^{p-1}\left|\nabla \beta v_{\varepsilon}\right|\right] \\
& +B_{1}\left[\int_{\Omega}\left|\alpha u_{1}\right|\left|\beta v_{\varepsilon}\right|^{p-1}+\left|\alpha u_{1}\right|^{p-1}\left|\beta v_{\varepsilon}\right|\right] \\
& +C_{1}\left[\int_{\Omega}\left|\alpha u_{1}\right| \frac{\left|\beta v_{\varepsilon}\right|^{q-1}}{|x|^{s}}+\left|\alpha u_{1}\right|^{q-1} \frac{\left|\beta v_{\varepsilon}\right|}{|x|^{s}}\right] \\
& \leq \frac{1}{p} \int_{\Omega}\left|\nabla\left(\alpha u_{1}\right)\right|^{p}-\frac{\lambda}{p} \int_{\Omega}\left|\alpha u_{1}\right|^{p}-\frac{1}{q} \int_{\Omega} \frac{\left|\alpha u_{1}\right|^{q}}{|x|^{s}} \\
& +\frac{1}{p} \int_{\Omega}\left|\nabla\left(\beta v_{\varepsilon}\right)\right|^{p}-\frac{\lambda}{p} \int_{\Omega}\left|\beta v_{\varepsilon}\right|^{p}-\frac{1}{q} \int_{\Omega} \frac{\left|\beta v_{\varepsilon}\right|^{q}}{|x|^{s}} \\
& +A_{2}\left(|\alpha|^{p}+|\beta|^{p}\right) \varepsilon^{\frac{n-p}{p(p-s)}}+B_{2}\left(|\alpha|^{p}+|\beta|^{p}\right) \varepsilon^{\frac{(n-p)(p-1)}{p(p-s)}} \\
& +C_{2}\left(|\alpha|^{q}+|\beta|^{q}\right) \varepsilon^{\frac{n-p}{p(p-s)}} \\
& \leq E_{\lambda, q}\left(\alpha u_{1}\right)+E_{\lambda}\left(\beta v_{\varepsilon}\right)+\frac{|\beta|^{p^{*}(s)}}{p^{*}(s)}-\frac{|\beta|^{q}}{q} \int_{\Omega} \frac{\left|v_{\varepsilon}\right|^{q}}{|x|^{s}} \\
& +A_{3} \varepsilon^{\frac{n-p}{p(p-s)}}+B_{3} \varepsilon^{\frac{(n-p)(p-1)}{p(p-s)}} \\
& \leq c_{1, q}+\frac{p-s}{p(n-s)} \mu_{s}^{\frac{n-s}{p-s}}+C \varepsilon^{\frac{n-p}{p-s}}+\frac{|\beta|^{p^{*}(s)}}{p^{*}(s)}-\frac{|\beta|^{q}}{q} \int_{\Omega} \frac{\left|v_{\varepsilon}\right|^{q}}{|x|^{s}}-O\left(\varepsilon^{\frac{p(p-1)}{p-s}}\right) \\
& +A_{3} \varepsilon^{\frac{n-p}{p(n-s)}}+B_{3} \varepsilon^{\frac{(n-p)(p-1)}{p(p-s)}} \\
& \leq c_{1, q}+\frac{p-s}{p(n-s)} \mu_{s}^{\frac{n-s}{p-s}}+C \varepsilon^{\frac{n-p}{p-s}}+A_{3} \varepsilon^{\frac{n-p}{p(p-s)}}+B_{3} \varepsilon^{\frac{(n p)(p-1)}{p(p-s)}} \\
& -C_{4} \varepsilon^{\frac{p(p-1)}{p-s}}+\frac{|\beta|^{p^{*}(s)}}{p^{*}(s)}-\frac{|\beta|^{q}}{q} \int_{\Omega} \frac{\left|v_{\varepsilon}\right|^{q}}{|x|^{s}} .
\end{aligned}
$$

Since $p^{3}-p^{2}+p<n$, we may choose $\varepsilon$ small enough so that

$$
C \varepsilon^{\frac{n-p}{p-s}}+A_{3} \varepsilon^{\frac{n-p}{p(p-s)}}+B_{3} \varepsilon^{\frac{(n-p)(p-1)}{p(p-s)}}-C_{4} \varepsilon^{\frac{p(p-1)}{p-s}} \leq-2 \sigma
$$

for some constant $\sigma>0$. Now choose $\delta_{0}>0$ small enough so that

$$
\frac{|\beta|^{p^{*}(s)}}{p^{*}(s)}-\frac{|\beta|^{q}}{q} \int_{\Omega} \frac{\left|v_{\varepsilon}\right|^{q}}{|x|^{s}}<\sigma \text { for } 0<\left|q-p^{*}(s)\right|<\delta_{0} .
$$

The proof of the lemma is now complete.

Proof of Theorem 9.1. In order to get the second solution, we shall consider the second solutions $u_{2, q}$ of the problems corresponding to $q<p^{*}(s)$ and we will find a 
limit as $q \rightarrow p^{*}(s)$. The location of $u_{2, q}$ on the dual sets $M_{2}^{q}$ will be crucial for the compactness.

Since $c_{2, q}$ is bounded uniformly in $q$, there is $K>0$ such that

$$
\left\|\nabla u_{2, q}\right\|_{p} \leq K \text { whenever } 0<\left|q-p^{*}(s)\right|<\delta_{0} .
$$

For $x \in \Omega$, define $\left(u_{2, q}\right)^{+}(x)=\max \left\{u_{2, q}(x), 0\right\}$ and $\left(u_{2, q}\right)^{-}(x)=\max \left\{-u_{2, q}(x), 0\right\}$. Since $u_{2, q} \in M_{2}^{q}$, both $\left(u_{2, q}\right)^{+}$and $\left(u_{2, q}\right)^{-}(x)$ are non-zero and belong to $H_{0}^{1, p}(\Omega)$. In addition,

$$
\left\|\nabla\left(u_{2, q}\right)^{ \pm}\right\| \leq K \text { whenever } 0<\left|q-p^{*}(s)\right|<\delta_{0} .
$$

Thus, we can find $q_{n}$ such that $q_{n} \rightarrow p^{*}(s)$ as $n \rightarrow+\infty, u^{+}, u^{-} \in H_{0}^{1, p}$ and

$$
\left(u_{2, q_{n}}\right)^{ \pm} \rightarrow u^{ \pm} \text {weakly in } H_{0}^{1, p} \text { as } n \rightarrow+\infty \text {. }
$$

We claim that $u^{+} \neq 0$ and $u^{-} \neq 0$. To shorten notation, set $u_{n}^{ \pm}=\left(u_{2, q_{n}}\right)^{ \pm}, c_{1, n}=$ $c_{1, q_{n}}, E_{n}=E_{\lambda, q_{n}}$ and $\Gamma_{n}=M_{1}^{q_{n}}$. Since $u_{n}$ is the solution of the corresponding sub-critical problem, we have that $u_{n}^{ \pm} \in \Gamma_{n}$. In particular,

$$
E_{n}\left(u_{n}^{ \pm}\right) \geq c_{1, n} .
$$

From Lemma 9.1, we also know that

$$
E_{n}\left(u_{n}^{+}\right)+E_{n}\left(u_{n}^{-}\right)=E_{n}\left(u_{n}\right)=c_{2, q_{n}} \leq c_{1, n}+\frac{p-s}{p(n-s)} \mu_{s}^{\frac{n-s}{p-s}}-\sigma
$$

for $n$ large. Necessarily,

$$
E_{n}\left(u_{n}^{ \pm}\right) \leq \frac{p-s}{p(n-s)} \mu_{s}^{\frac{n-s}{p-s}}-\sigma
$$

for $\mathrm{n}$ large. From the fact that $u_{n}^{ \pm} \in \Gamma_{n}$ and $c_{1}^{n} \rightarrow c_{1}>0$, we derive

$$
K_{1} \leq \int_{\Omega} \frac{\left|u_{n}^{ \pm}\right|^{q_{n}}}{|x|^{s}} \leq K_{2}
$$

with suitable positive constants $K_{1}$ and $K_{2}$.

Arguing by contradiction, assume, for example, that $u^{+}=0$. From the above and the fact that $u_{n}^{ \pm} \in \Gamma_{n}$, we obtain

and

$$
\frac{1}{p}\left\|\nabla u_{n}^{+}\right\|_{p}^{p}-\frac{1}{q_{n}} \int_{\Omega} \frac{\left|u_{n}^{+}\right|^{q_{n}}}{|x|^{s}} \leq \frac{p-s}{p(n-s)} \mu_{s}^{\frac{n-s}{p-s}}-\sigma+o(1)
$$

Consequently,

$$
\left\|\nabla u_{n}^{+}\right\|_{p}^{p}-\int_{\Omega} \frac{\left|u_{n}^{+}\right|^{q_{n}}}{|x|^{s}}=o(1)
$$

$$
\begin{aligned}
& \mu_{s}\left(\int_{\Omega} \frac{\left|u_{n}^{+}\right|^{p^{*}(s)}}{|x|^{s}}\right)^{\frac{p}{p^{*}(s)}} \\
& \quad \leq\left\|\nabla u_{n}^{+}\right\|_{p}^{p}=\int_{\Omega} \frac{\left|u_{n}^{+}\right|^{q_{n}}}{|x|^{s}}+o(1)=\int_{\Omega} \frac{\left|u_{n}^{+}\right|^{q_{n}}}{|x|^{\frac{q_{n}}{p^{*}(s)}}} \cdot \frac{1}{|x|^{s\left(1-\frac{q_{n}}{p^{*}(s)}\right)}} \\
& \quad \leq\left(\int_{\Omega} \frac{\left|u_{n}^{+}\right|^{q_{n}}}{|x|^{s}}\right)^{\frac{q_{n}}{p^{*}(s)}}\left(\int_{\Omega} \frac{1}{|x|^{s}}\right)^{\frac{p^{*}(s)-q_{n}}{p^{*}(s)}}+o(1) .
\end{aligned}
$$

Since $\int_{\Omega} \frac{\left|u_{n}^{+}\right|^{q_{n}}}{|x|^{s}}$ is bounded away from zero, we conclude that

$$
\left(\int_{\Omega} \frac{\left|u_{n}^{+}\right|^{q_{n}}}{|x|^{s}}\right)^{\frac{q_{n}-p}{p^{*}(s)}} \geq\left(\int_{\Omega} \frac{1}{|x|^{s}}\right)^{\frac{q_{n}-p^{*}(s)}{p^{*}(s)}} \mu_{s}+o(1) .
$$


That is,

Thus, we have

$$
\int_{\Omega} \frac{\left|u_{n}^{+}\right|^{q_{n}}}{|x|^{s}} \geq \mu_{s}^{\frac{n-s}{p-s}}+o(1)
$$

$$
\begin{aligned}
& \frac{p-s}{p(n-s)} \mu_{s}^{\frac{n-s}{p-s}}+o(1) \leq \frac{p-s}{p(n-s)} \int_{\Omega} \frac{\left|u_{n}^{+}\right|^{q_{n}}}{|x|^{s}} \\
& \quad=\frac{1}{p}\left\|\nabla u_{n}^{+}\right\|_{p}^{p}-\frac{1}{q_{n}} \int_{\Omega} \frac{\left|u_{n}^{+}\right|^{q_{n}}}{|x|^{s}}+o(1) \leq \frac{p-s}{p(n-s)} \mu_{s}^{\frac{n-s}{p-s}}-\sigma+o(1) .
\end{aligned}
$$

This is a contradiction, and we conclude that $u^{+} \neq 0$. Similarly, $u^{-} \neq 0$.

Set $u=u^{+}-u^{-}$; that is, $u$ changes $\operatorname{sign}$ in $\Omega$ and

$$
u_{n}:=u_{q_{n}} \rightarrow u \text { weakly in } H_{0}^{1, p}(\Omega) .
$$

So, $\left\langle E_{\lambda}^{\prime}(u), w\right\rangle=0$ for any $w \in H_{0}^{1, p}(\Omega)$, i.e. $u$ is a weak solution of $\left(P_{\lambda}\right)$. Now, we prove that a subsequence of $\left\{u_{n}\right\}$ converges to $u$ strongly in $H_{0}^{1, p}(\Omega)$ and conclude that $u$ is a solution of $\left(\mathrm{P}_{\lambda, p^{*}(s)}\right)$ that is located on $M_{2}$.

Since $\left\{E\left(u_{n}\right)\right\}$ is bounded and $E_{n}^{\prime}\left(u_{n}\right) \rightarrow 0$, we may assume that the conclusions of Lemma 4.4 hold for the sequence $\left(u_{n}\right)_{n}$.

Note that $u \in M_{1}$; hence $E(u) \geq c_{1}$. Set $u_{n}=u+w_{n}$, with $w_{n} \rightarrow 0$ weakly in $H_{0}^{1, p}$. We have

$$
\begin{aligned}
c_{1, n} & +\frac{p-s}{p(n-s)} \mu_{s}^{\frac{n-s}{p-s}}-\sigma \geq E_{n}\left(u+w_{n}\right) \\
& \geq \frac{1}{p}\|\nabla u\|_{p}^{p}-\frac{1}{q_{n}} \int_{\Omega} \frac{|u|^{q_{n}}}{|x|^{s}}-\frac{\lambda}{r}\|u\|_{r}^{r}+\frac{1}{p}\left\|\nabla w_{n}\right\|_{p}^{p}-\frac{1}{q_{n}} \int_{\Omega} \frac{\left|w_{n}\right|^{q_{n}}}{|x|^{s}}+o(1) \\
& \geq c_{1}+\frac{1}{p}\left\|\nabla w_{n}\right\|_{p}^{p}-\frac{1}{q_{n}} \frac{\left|w_{n}\right|^{q_{n}}}{|x|^{s}}+o(1) .
\end{aligned}
$$

Since $\left|c_{1, n}-c_{1}\right|=o(1)$, we derive

$$
\frac{1}{p}\left\|\nabla w_{n}\right\|_{p}^{p}-\frac{1}{q_{n}} \int_{\Omega} \frac{\left|w_{n}\right|^{q_{n}}}{|x|^{s}} \leq \frac{p-s}{p(n-s)} \mu_{s}^{\frac{n-s}{p-s}}-\sigma+o(1) .
$$

Furthermore,

i.e.

$$
0=\left\langle E_{n}^{\prime}\left(u_{n}\right), u_{n}\right\rangle=\left\langle E^{\prime}(u), u\right\rangle+\left\|\nabla w_{n}\right\|_{p}^{p}-\int_{\Omega} \frac{\left|w_{n}\right|^{q_{n}}}{|x|^{s}}+o(1)
$$

$$
\left\|\nabla w_{n}\right\|_{p}^{p}-\int_{\Omega} \frac{\left|w_{n}\right|^{q_{n}}}{|x|^{s}}=o(1) .
$$

The last two relations show that the sequence $\left\|\nabla w_{n}\right\|_{p}$ cannot be bounded away from zero, and therefore a subsequence of $\left\{w_{n}\right\}$ converges strongly to zero.

\section{Sobolev CRITICAL NON-SINGUlar TERM}

In this section, we prove Theorem 1.4. We reformulate it as follows.

Theorem 10.1. Suppose $1<p \leq q<p^{*}(s)$ and $r=p^{*}$ in the equation

$$
\left(P_{\lambda, \mu}\right) \quad\left\{\begin{aligned}
-\triangle_{p} u & =\lambda|u|^{p^{*}-2} u+\mu \frac{|u|^{q-2}}{|x|^{s}} u \quad \text { in } \Omega, \\
\left.u\right|_{\partial \Omega} & =0 .
\end{aligned}\right.
$$

Then $\left(\mathrm{P}_{\lambda, \mu}\right)$ has a positive solution if any one of the following conditions holds: 
(1) $p<q, n>\frac{p(p-1)(q-s)+p^{2}}{p+(p-1)(q-p)}$ and $\lambda>0, \mu>0$.

(2) $p=q, n \geq p^{2}-(p-1) s$ and $\lambda>0, \mu_{s, p}>\mu>0$.

If one of the following conditions holds:

(1') $p<q, n>\frac{p(p-1)(q-s)+p}{1+(p-1)(q-p)}$ and $\lambda>0, \mu>0$,

(2') $p=q, n>p((p-1)(p-s)+1)$ and $\lambda>0, \mu_{s, p}>\mu>0$,

then $\left(\mathrm{P}_{\lambda, \mu}\right)$ has also a sign-changing solution.

Remark 10.1. The existence of a positive solution under condition (2) above has already been noticed in [13] in the case where $p=q$.

By scaling, we can always assume that $\lambda=1$. The corresponding functional is again

$$
E_{\mu}(u)=\frac{1}{p} \int_{\Omega}|\nabla u|^{p}-\frac{1}{p^{*}} \int_{\Omega}|u|^{p^{*}}-\frac{\mu}{q} \int_{\Omega} \frac{|u|^{q}}{|x|^{s}} .
$$

Recall that under any one of the above conditions, the set

$$
M_{1}=\left\{u \in H_{0}^{1, p}(\Omega) ; u \neq 0,\left\langle E_{\mu}^{\prime}(u), u\right\rangle=0\right\}
$$

is dual to the class

$$
\mathcal{F}_{1}=\left\{\gamma \in C\left([0,1] ; H_{0}^{1, p}(\Omega)\right) ; \gamma(0)=0, \gamma(1) \neq 0 \text { and } E_{\mu}(\gamma(1)) \leq 0\right\} .
$$

Moreover, the energy level $c_{1}=\inf _{A \in \mathcal{F}_{1}} \sup _{u \in A} E_{\mu}(u)$ is equal to $\inf _{u \in M_{1}} E_{\mu}(u)$.

By Theorem 4.1.(3), $E_{\mu}$ satisfies (PS) $)_{c}$ for any $c<\frac{1}{n} \mu_{0} \frac{n}{p}$. So, the existence of the first positive solution follows immediately from the following estimates.

Lemma 10.1. If $r=p^{*}$, then $c_{1}<\frac{1}{n} \mu_{0}^{\frac{n}{p}}$ in any one of the following three cases:

(1) $q=p, 0<\mu<\mu_{s, p}$ and $n \geq p^{2}-(p-1) s$.

(2) $p<q<p^{*}(s)$ and $\mu$ large enough.

(3) $p<q<p^{*}(s)$ and $n>\frac{p(p-1)(q-s)+p^{2}}{p+(p-1)(q-p)}$.

Proof. Take $v_{\varepsilon}$ to be the function as in Lemma 11.2 of the appendix. Then, as in the proof of Lemma 9.1, we consider:

Case $q>p$. We have

$$
\begin{aligned}
& \max _{0 \leq t<\infty} E_{\mu}\left(t v_{\varepsilon}\right) \leq \frac{1}{n} \mu_{0}^{\frac{n}{p}}+O\left(\varepsilon^{\frac{n-p}{p}}\right)-\frac{\mu}{q}\left(\frac{\mu_{0}}{s}\right)^{\frac{q}{p^{*}-p}} \int_{\Omega} \frac{\left|v_{\varepsilon}\right|^{q}}{|x|^{s}} \\
& =\frac{1}{n} \mu_{0}^{\frac{n}{p}}+O\left(\varepsilon^{\frac{n-p}{p}}\right)-O\left(\varepsilon^{\frac{(n-p)(p-1)}{p^{2}}\left(p^{*}(s)-q\right)}\right) .
\end{aligned}
$$

where we require that $q>\frac{n-s}{n-p}(p-1)$. The estimate then follows.

Case $q=p$. We have

$$
\begin{aligned}
\max _{0 \leq t<\infty} & E_{\mu}\left(t v_{\varepsilon}\right)=\frac{1}{n}\left(\int_{\Omega}\left|\nabla v_{\varepsilon}\right|^{p}-\frac{\left|v_{\varepsilon}\right|^{p}}{|x|^{s}}\right)^{\frac{n}{p}} \\
& = \begin{cases}\frac{1}{n} \mu_{0}^{\frac{n}{p}}+O\left(\varepsilon^{\frac{n-p}{p}}\right)-O\left(\varepsilon^{\frac{(n-p)(p-1)}{p^{2}}\left(p^{*}(s)-p\right)}\right), & p>\frac{n-s}{n-p}(p-1), \\
\frac{1}{n} \mu_{0}^{\frac{n}{p}}+O\left(\varepsilon^{\frac{n-p}{p}}\right)-O\left(\varepsilon^{\frac{n-p}{p}}|\log \varepsilon|\right), & p=\frac{n-s}{n-p}(p-1) .\end{cases}
\end{aligned}
$$

Since

the conclusions now follow immediately.

$$
\frac{(n-p)(p-1)}{p^{2}}\left(p^{*}(s)-p\right)=\frac{(p-s)(p-1)}{p},
$$


For the sign changing solution, we shall proceed as in the case of the HardySobolev critical exponent. First we find appropriate sign changing solutions for the sub-critical problem, i.e. when $r<p^{*}$, and then we pass to the limit as $r \rightarrow p^{*}$.

Write again

$$
\begin{gathered}
E_{\mu, r}(u)=\frac{1}{p} \int_{\Omega}|\nabla u|^{p}-\frac{1}{r} \int_{\Omega}|u|^{r}-\frac{\mu}{q} \int_{\Omega} \frac{|u|^{q}}{|x|^{s}}, \\
\mathcal{F}_{1}^{r}=\left\{\gamma \in C\left([0,1] ; H_{0}^{1, p}(\Omega)\right) ; \gamma(0)=0, \gamma(1) \neq 0 \text { and } E_{\mu, r}(\gamma(1)) \leq 0\right\}, \\
M_{1}^{r}=\left\{u \in H_{0}^{1, p}(\Omega) ; u \neq 0,\left\langle\left(E_{\mu, r}\right)^{\prime}(u), u\right\rangle=0\right\},
\end{gathered}
$$

and

$$
c_{1, r}=\inf _{A \in \mathcal{F}_{1}} \sup _{u \in A} E_{\mu, r}(u) .
$$

Then, as previously, we know that $M_{1}^{r}$ is dual to $\mathcal{F}_{1}^{r}$ and $c_{1, r}=\inf _{u \in M_{1}} E_{\mu}(u)$. Also define

$$
c_{2, r}=\inf _{A \in \mathcal{F}_{2}} \sup _{u \in A} E_{\mu, r}(u),
$$

where $\mathcal{F}_{2}$ is defined in section 5 . We write $c_{2}\left(\operatorname{resp} . E_{\mu}\right)$ for $c_{2, p^{*}}\left(\operatorname{resp} E_{\mu, p^{*}}\right)$.

Lemma 10.2. Under either one of the following conditions,

(1) $p=q, 0<\mu<\mu_{s, p}$ and $n>p(p-1)(p-s)+p$,

(2) $p<q, \mu>0$ and $n>\frac{p(p-1)(q-s)+p}{1+(p-1)(q-p)}$,

we have

(i) $c_{i, r} \rightarrow c_{i}(i=1,2)$ as $r \rightarrow p^{*}$,

(ii) $c_{2, r} \leq c_{1, r}+\frac{1}{n} \mu_{0}^{\frac{n}{p}}-\sigma$ for some $\sigma>0$ and $r$ sufficiently close to $p^{*}$.

Proof. For the first conclusion, the proof is exactly the same as in the last section. For the second one, we can assume that the first solution $u_{1}$ is smooth and $\nabla u_{1} \in$ $L^{\infty}(\Omega)$.

For $\varepsilon>0$ and $q$ sufficiently close to $p^{*}$, apply the calculus lemma to obtain

$$
\begin{aligned}
E_{\mu, r}\left(\alpha u_{1}+\beta v_{\varepsilon}\right) \leq & E_{\mu, r}\left(\alpha u_{1}\right)+E_{\mu, r}\left(\beta v_{\varepsilon}\right) \\
& +A_{1}\left[\int_{\Omega}\left|\nabla \alpha u_{1}\right|\left|\nabla \beta v_{\varepsilon}\right|^{p-1}+\left|\nabla \alpha u_{1}\right|^{p-1}\left|\nabla \beta v_{\varepsilon}\right|\right] \\
& +B_{1}\left[\int_{\Omega}\left|\alpha u_{1}\right|\left|\beta v_{\varepsilon}\right|^{r-1}+\left|\alpha u_{1}\right|^{r-1}\left|\beta v_{\varepsilon}\right|\right] \\
& +C_{1}\left[\int_{\Omega}\left|\alpha u_{1}\right| \frac{\left|\beta v_{\varepsilon}\right|^{q-1}}{|x|^{s}}+\left|\alpha u_{1}\right|^{q-1} \frac{\left|\beta v_{\varepsilon}\right|}{|x|^{s}}\right] \\
\leq & E_{\mu, r}\left(\alpha u_{1}\right)+E_{\mu, r}\left(\beta v_{\varepsilon}\right)+A_{2}\left(|\alpha|^{p}+|\beta|^{p}\right) \varepsilon^{\frac{n-p}{p^{2}}} \\
& +B_{2}\left(|\alpha|^{r}+|\beta|^{r}\right) \varepsilon^{\frac{p-1}{p}\left(n-\frac{(r-1)(n-p)}{p}\right)} \\
& +C_{2}\left(|\alpha|^{q}+|\beta|^{q}\right) \varepsilon^{\frac{n-p}{p^{2}}} .
\end{aligned}
$$

Again, we note that for $\varepsilon$ sufficiently small,

$$
\lim _{\alpha, \beta \rightarrow \infty} E_{\mu, r}\left(\alpha u_{1}+\beta v_{\varepsilon}\right)=-\infty .
$$

So we may assume that $\alpha$ and $\beta$ are in a bounded set.

Case 1. Assume $p<q, 0<\mu$ and $n>\frac{p(p-1)(q-s)+p}{1+(p-1)(q-p)}$. 
Then, by the calculus lemma,

$$
\begin{aligned}
& E_{\mu, r}\left(\alpha u_{1}+\beta v_{\varepsilon}\right) \\
& \leq E_{\mu, r}\left(\alpha u_{1}\right)+E_{\mu}\left(\beta v_{\varepsilon}\right)+\frac{|\beta|^{p^{*}}}{p^{*}}-\frac{|\beta|^{r}}{r} \int_{\Omega}\left|v_{\varepsilon}\right|^{r} \\
&+A_{3} \varepsilon^{\frac{n-p}{p^{2}}}+B_{3} \varepsilon^{\frac{p-1}{p}\left(n-\frac{(r-1)(n-p)}{p}\right)} \\
& \leq c_{1, r}+\frac{1}{n} S^{\frac{n}{p}}+C \varepsilon^{\frac{n-p}{p}}+\frac{|\beta|^{p^{*}}}{p^{*}}-\frac{|\beta|^{r}}{r} \int_{\Omega}\left|v_{\varepsilon}\right|^{r}-\frac{\mu}{q}\left(\frac{S}{2}\right)^{\frac{q}{p^{*}-p}} \int_{\Omega} \frac{\left|v_{\varepsilon}\right|^{q}}{|x|^{s}} \\
&+A_{3} \varepsilon^{\frac{n-p}{p^{2}}}+B_{3} \varepsilon^{\frac{p-1}{p}\left(n-\frac{(r-1)(n-p)}{p}\right)} \\
& \leq c_{1, r}+\frac{1}{n} S^{\frac{n}{p}}+C \varepsilon^{\frac{n-p}{p}}+A_{3} \varepsilon^{\frac{n-p}{p^{2}}}+B_{3} \varepsilon^{\frac{p-1}{p}\left(n-\frac{(r-1)(n-p)}{p}\right)} \\
&-C_{4} \varepsilon^{\frac{(n-p)(p-1)}{p^{2}}\left(p^{*}(s)-q\right)}+\frac{|\beta|^{p^{*}}}{p^{*}}-\frac{|\beta|^{r}}{r} \int_{\Omega}\left|v_{\varepsilon}\right|^{r},
\end{aligned}
$$

where we require $q>\frac{n-s}{n-p}(p-1)$. From

$$
\frac{p-1}{p}\left(n-\frac{(r-1)(n-p)}{p}\right)>\frac{(n-p)(p-1)}{p^{2}}\left(p^{*}(s)-q\right)
$$

we get $q>r-1-\frac{n s}{n-p}$. From

$$
\frac{n-p}{p^{2}}>\frac{(n-p)(p-1)}{p^{2}}\left(p^{*}(s)-q\right),
$$

we get that $q>p^{*}(s)-\frac{1}{p-1}$. Since

$$
p^{*}(s)-\frac{1}{p-1} \geq p^{*}(s)-1=p^{*}-1-\frac{p s}{n-p}>r-1-\frac{n s}{n-p},
$$

the hypothesis $q>p^{*}(s)-\frac{1}{p-1}$ (i.e. $\left.n>\frac{p(p-1)(q-s)+p}{1+(p-1)(q-p)}\right)$ is sufficient to allow us to choose $\varepsilon$ small enough so that

$$
C \varepsilon^{\frac{n-p}{p}}+A_{3} \varepsilon^{\frac{n-p}{p^{2}}}+B_{3} \varepsilon^{\frac{p-1}{p}\left(n-\frac{(r-1)(n-p)}{p}\right)}-C_{4} \varepsilon^{\frac{(n-p)(p-1)}{p^{2}}\left(p^{*}(s)-q\right)} \leq-2 \sigma
$$

for some constant $\sigma>0$. Now choose $\delta_{0}>0$ small enough so that

$$
\frac{|\beta|^{p^{*}}}{p^{*}}-\frac{|\beta|^{r}}{r} \int_{\Omega}\left|v_{\varepsilon}\right|^{r}<\sigma \text { for } 0<\left|r-p^{*}\right|<\delta_{0}
$$

Thus we have proved the case for $q>p$.

Case 2. $q=p, n>p(p-1)(p-s)+p$ and $0<\mu<\mu_{s, p}$. 
We assume that $\alpha$ and $\beta$ are in a bounded set, and we estimate $E_{\lambda}\left(\alpha u_{1}+\beta v_{\varepsilon}\right)$ :

$$
\begin{aligned}
E_{\mu, r}(\alpha & \left.u_{1}+\beta v_{\varepsilon}\right) \\
\leq & E_{\mu, r}\left(\alpha u_{1}\right)+E_{\mu, r}\left(\beta v_{\varepsilon}\right) \\
& +A_{1}\left[\int_{\Omega}\left|\nabla \alpha u_{1}\right|\left|\nabla \beta v_{\varepsilon}\right|^{p-1}+\left|\nabla \alpha u_{1}\right|^{p-1}\left|\nabla \beta v_{\varepsilon}\right|\right] \\
& +B_{1}\left[\int_{\Omega}\left|\alpha u_{1}\right| \frac{\left|\beta v_{\varepsilon}\right|^{p-1}}{|x|^{s}}+\left|\alpha u_{1}\right|^{p-1} \frac{\left|\beta v_{\varepsilon}\right|}{|x|^{s}}\right] \\
& +C_{1}\left[\int_{\Omega}\left|\alpha u_{1}\right|\left|\beta v_{\varepsilon}\right|^{r-1}+\left|\alpha u_{1}\right|^{r-1}\left|\beta v_{\varepsilon}\right|\right] \\
\leq & E_{\mu, r}\left(\alpha u_{1}\right)+E_{\mu}\left(\beta v_{\varepsilon}\right)+\frac{|\beta|^{p^{*}}}{p^{*}}-\frac{|\beta|^{r}}{r} \int_{\Omega}\left|v_{\varepsilon}\right|^{r} \\
& +A_{3} \varepsilon^{\frac{n-p}{p^{2}}}+B_{3} \varepsilon^{\frac{p-1}{p}\left(n-\frac{(r-1)(n-p)}{p}\right)} \\
\leq & c_{1, r}+\frac{1}{n} S^{\frac{n}{p}}+C \varepsilon^{\frac{n-p}{p}}+\frac{|\beta|^{p^{*}}}{p^{*}}-\frac{|\beta|^{r}}{r} \int_{\Omega}\left|v_{\varepsilon}\right|^{r}-O\left(\varepsilon^{\frac{(p-s)(p-1)}{p}}\right) \\
& +A_{3} \varepsilon^{\frac{n-p}{p^{2}}}+B_{3} \varepsilon^{\frac{p-1}{p}\left(n-\frac{(r-1)(n-p)}{p}\right)} \\
\leq & c_{1, r}+\frac{1}{n} S^{\frac{n}{p}}+C \varepsilon^{\frac{n-p}{p}}+A_{3} \varepsilon^{\frac{n-p}{p^{2}}}+B_{3} \varepsilon^{\frac{p-1}{p}\left(n-\frac{(r-1)(n-p)}{p}\right)} \\
& -C_{4} \varepsilon^{\frac{(p-s)(p-1)}{p}}+\frac{|\beta|^{p^{*}}}{p^{*}}-\frac{|\beta|^{r}}{r} \int_{\Omega}\left|v_{\varepsilon}\right|^{r} .
\end{aligned}
$$

Note that we have required that $p>\frac{n-s}{n-p}(p-1)$. By the assumption, we can choose $\varepsilon$ small enough so that

$$
C \varepsilon^{\frac{n-p}{p}}+A_{3} \varepsilon^{\frac{n-p}{p^{2}}}+B_{3} \varepsilon^{\frac{p-1}{p}\left(n-\frac{(r-1)(n-p)}{p}\right)}-C_{4} \varepsilon^{\frac{(p-s)(p-1)}{p}} \leq-2 \sigma
$$

for some constant $\sigma>0$. Now choose $\delta_{0}>0$ small enough so that

$$
\frac{|\beta|^{p^{*}}}{p^{*}}-\frac{|\beta|^{r}}{r} \int_{\Omega}\left|v_{\varepsilon}\right|^{r}<\sigma \text { for } 0<\left|q-p^{*}\right|<\delta_{0} .
$$

The proof of this lemma is now complete.

The rest of the proof of the theorem is now very similar to Theorem 9.1. The details are left for the interested reader.

\section{Appendix: Estimates on the extremal Sobolev-Hardy functions}

Assume, without loss of generality, that $0 \in \Omega$, and let

$$
U_{\varepsilon}(x)=\left(\varepsilon+|x|^{\frac{p-s}{p-1}}\right)^{\frac{p-n}{p-s}} .
$$

$U_{\varepsilon}(x)$ is a function in $H^{1, p}\left(\mathbf{R}^{n}\right)$ where the best constant in the Sobolev-Hardy inequality is attained. They are, modulo translation and dilations, the unique positive ones where the best constant is achieved. (See section 2.)

Let $0 \leq \phi(x) \leq 1$ be a function in $C_{0}^{\infty}(\Omega)$ defined as

$$
\phi(x)= \begin{cases}1 & \text { if }|x| \leq R, \\ 0 & \text { if }|x| \geq 2 R,\end{cases}
$$

where $B_{2 R}(0) \subset \Omega$. Set $u_{\varepsilon}(x)=\phi(x) U_{\varepsilon}(x)$. For $\varepsilon \rightarrow 0$, the behavior of $u_{\varepsilon}$ has to be the same as that of $U_{\varepsilon}$ but we need precise estimates of the error terms. 
Lemma 11.1. Assume $0 \leq s<p, p \geq 2$ and $q=\frac{n-s}{n-p} p$. By taking

$$
v_{\varepsilon}=\frac{u_{\varepsilon}}{\left(\int_{\Omega} \frac{\left|u_{\varepsilon}\right|^{q}}{|x|^{s}}\right)^{\frac{1}{q}}}
$$

so that $\int_{\Omega} \frac{\left|v_{\varepsilon}\right|^{q}}{|x|^{s}}=1$, we have the following estimates:

(1) $\left\|\nabla v_{\varepsilon}\right\|_{p}^{p}=\mu_{s}+O\left(\varepsilon^{\frac{n-p}{p-s}}\right)$,

(2) $\int_{\Omega}\left|\nabla v_{\varepsilon}\right|^{\alpha}=O\left(\varepsilon^{\frac{(n-p) \alpha}{p(p-s)}}\right)$, for $\alpha=1,2, p-2, p-1$.

(3) if $r>p^{*}\left(1-\frac{1}{p}\right)$, then

$$
C_{1} \varepsilon^{\left(\frac{p-1}{p-s}\right)\left(n-\frac{r(n-p)}{p}\right)} \leq\left\|v_{\varepsilon}\right\|_{r}^{r} \leq C_{2} \varepsilon^{\left(\frac{p-1}{p-s}\right)\left(n-\frac{r(n-p)}{p}\right)},
$$

(4) if $r=p^{*}\left(1-\frac{1}{p}\right)$, then

$$
C_{1} \varepsilon^{\frac{(n-p) r}{p(p-s)}}|\log \varepsilon| \leq\left\|v_{\varepsilon}\right\|_{r}^{r} \leq C_{2} \varepsilon^{\frac{(n-p) r}{p(p-s)}}|\log \varepsilon|,
$$

(5) if $r<p^{*}\left(1-\frac{1}{p}\right)$, then

$$
C_{1} \varepsilon^{\frac{(n-p) r}{p(p-s)}} \leq\left\|v_{\varepsilon}\right\|_{r}^{r} \leq C_{2} \varepsilon^{\frac{(n-p) r}{p(p-s)}},
$$

(6) if $p<r<p^{*}$, then $\left\|v_{\varepsilon}\right\|_{r}^{r} \rightarrow 0$ (as $\varepsilon \rightarrow 0$ ),

$$
\int_{\Omega} \frac{\left|v_{\varepsilon}\right|^{q-1}}{|x|^{s}}=O\left(\varepsilon^{\frac{p-1}{p}\left(\frac{n-p}{p-s}\right)}\right)
$$

$$
\int_{\Omega} \frac{\left|v_{\varepsilon}\right|}{|x|^{s}}=O\left(\varepsilon^{\frac{n-p}{p(p-s)}}\right)
$$

where $C_{1}, C_{2}>0$ are constants.

Proof. Let

$$
k(\varepsilon)=\left(\varepsilon \cdot(n-s)\left(\frac{n-p}{p-1}\right)^{p-1}\right)^{\frac{n-p}{p(p-s)}} .
$$

Then $y_{\varepsilon}(x)=k(\varepsilon) U_{\varepsilon}(x)$ is the extremal function in the Sobolev-Hardy inequality. Furthermore,

$$
k(\varepsilon)^{p}\left\|\nabla U_{\varepsilon}(x)\right\|_{p}^{p}=\left\|\nabla y_{\varepsilon}(x)\right\|_{p}^{p}=\mu_{s}^{\frac{n-s}{p-s}}
$$

The gradient of $u_{\varepsilon}(x)$ is given by

$$
\begin{aligned}
\nabla u_{\varepsilon}(x) & =\left(\varepsilon+|x|^{\frac{p-s}{p-1}}\right)^{\frac{p-n}{p-s}} \nabla \phi(x)+\frac{p-n}{p-1} \cdot \frac{x \phi(x)}{\left(\varepsilon+|x|^{\frac{p-s}{p-1}}\right)^{\frac{n-s}{p-s}}|x|^{\frac{p-2}{p-1}}} \\
& = \begin{cases}\frac{p-n}{p-1} \cdot \frac{x}{\left(\varepsilon+|x|^{\frac{p-s}{p-1}}\right)^{\frac{n-s}{p-s}}|x|^{\frac{p-2}{p-1}}} & \text { if }|x| \leq R, \\
0 & \text { if }|x| \geq 2 R .\end{cases}
\end{aligned}
$$

Thus we have

$$
\begin{aligned}
\int_{\Omega}\left|\nabla u_{\varepsilon}\right|^{p} & =O(1)+\int_{|x| \leq R}\left|\nabla U_{\varepsilon}(x)\right|^{p} d x=O(1)+\int_{\mathbf{R}^{n}}\left|\nabla U_{\varepsilon}(x)\right|^{p} d x \\
& =O(1)+\left\|\nabla U_{\varepsilon}(x)\right\|_{p}^{p},
\end{aligned}
$$


and

$$
\int_{\Omega} \frac{\left|u_{\varepsilon}\right|^{p}}{|x|^{s}}=O(1)+\int_{\mathbf{R}^{n}} \frac{\left|U_{\varepsilon}\right|^{q}}{|x|^{s}}=O(1)+\int_{\mathbf{R}^{n}} \frac{\left|y_{\varepsilon}\right|^{q}}{|x|^{s}} \cdot k^{-q}(\varepsilon)=O\left(k^{-q}(\varepsilon)\right) .
$$

From this we further get

$$
\begin{aligned}
\left\|\nabla v_{\varepsilon}\right\|_{p}^{p} & =\frac{\left\|\nabla u_{\varepsilon}\right\|_{p}^{p}}{\left(\int_{\Omega} \frac{\left|u_{\varepsilon}\right|^{q}}{|x|^{s}}\right)^{\frac{p}{q}}}=\frac{O(1)+\left\|\nabla U_{\varepsilon}\right\|_{p}^{p}}{\left(\int_{\Omega} \frac{\left|u_{\varepsilon}\right|^{q}}{|x|^{s}}\right)^{\frac{p}{q}}} \\
& =\frac{O(1)+\mu_{s}^{\frac{n-s}{p-s}} k(\varepsilon)^{-p}}{\left(\int_{\Omega} \frac{\left|u_{\varepsilon}\right|^{q}}{|x|^{s}}\right)^{\frac{p}{q}}}=\frac{O(1)+\mu_{s}^{\frac{n-s}{p-s}} k(\varepsilon)^{-p}}{O(1)+k(\varepsilon)^{-p} \mu_{s}^{\frac{p(n-s)}{(p-s) q}}} \\
& =O\left(k(\varepsilon)^{p}\right)+\mu_{s}^{\frac{n-s}{p-s}-\frac{p(n-s)}{(p-s) q}}=\mu_{s}+O\left(\varepsilon^{\frac{n-p}{p-s}}\right) .
\end{aligned}
$$

(1) is thus proved. For $(2)$, let $\omega_{n}$ denote the surface area of the $(n-1)$-sphere $S^{n-1}$ in $\mathbf{R}^{n}$; then

$$
\begin{aligned}
\int_{\Omega}\left|\nabla u_{\varepsilon}\right|^{\alpha} & =O(1)+\int_{|x| \leq R}\left(\frac{n-p}{p-1}\right)^{\alpha} \frac{|x|^{\alpha}}{\left(\varepsilon+|x|^{\frac{p-s}{p-1}}\right)^{\frac{\alpha(n-s)}{p-s}}|x|^{\frac{(p-2) \alpha}{p-1}}} d x \\
& =O(1)+\omega_{n} \int_{0}^{R}\left(\frac{n-p}{p-1}\right)^{\alpha} \frac{r^{\alpha} \cdot r^{n-1}}{\left(\varepsilon+r^{\frac{p-s}{p-1}}\right)^{\frac{\alpha(n-s)}{p-s}} r^{\frac{(p-2) \alpha}{p-1}}} d r \\
& \leq O(1)+\omega_{n} \int_{0}^{R}\left(\frac{n-p}{p-1}\right)^{\alpha} r^{\alpha+n-1-\frac{\alpha(n-s)}{p-1}-\frac{\alpha(p-2)}{p-1}} d r
\end{aligned}
$$

and the order of $r$ in the integrand is

$$
\begin{aligned}
\alpha+n-1-\frac{\alpha(n-s)}{p-1}-\frac{\alpha(p-2)}{p-1} & =\frac{p n-p-n+1-\alpha n+\alpha s+\alpha}{p-1} \\
& =\frac{p n-n-\alpha n+\alpha s+\alpha}{p-1}-1>-1
\end{aligned}
$$

for $\alpha=1, p-2, p-1$ and $\alpha=2$ if $p \geq 3$. Thus

$$
\int_{\Omega}\left|\nabla u_{\varepsilon}\right|^{\alpha}=O(1)
$$

and we conclude that

$$
\int_{\Omega}\left|\nabla v_{\varepsilon}\right|^{\alpha}=O\left(\varepsilon^{\frac{(n-p) \alpha}{p(p-s)}}\right)
$$

For (3), (4) and (5),

$$
\begin{aligned}
\left\|u_{\varepsilon}\right\|_{r}^{r} & =O(1)+\omega_{n} \int_{0}^{R}\left(\varepsilon+x^{\frac{p-s}{p-1}}\right)^{-\frac{n-p}{p-s} r} x^{n-1} d x \\
& =O(1)+\omega_{n} \varepsilon^{-\frac{n-p}{p-s} r+\frac{p-1}{p-s} n} \int_{0}^{R \varepsilon^{-\frac{p-1}{p-s}}}\left(1+x^{\frac{p-s}{p-1}}\right)^{-\frac{n-p}{p-s} r} x^{n-1} d x
\end{aligned}
$$


If $r=p^{*}\left(1-\frac{1}{p}\right)$, then $-\frac{n-p}{p-s} r+\frac{p-1}{p-s} n=0$, and

$$
\begin{aligned}
\left\|u_{\varepsilon}\right\|_{r}^{r} & =O(1)+\omega_{n} \int_{0}^{R \varepsilon^{-\frac{p-1}{p-s}}}\left(1+x^{\frac{p-s}{p-1}}\right)^{-\frac{n-p}{p-s} r} x^{n-1} d x \\
& =O(1)+\omega_{n} \int_{0}^{R \varepsilon^{-\frac{p-1}{p-s}}} \frac{1}{x} d x \\
& =O(1)+O(|\log \varepsilon|) .
\end{aligned}
$$

So we get

$$
\left\|v_{\varepsilon}\right\|_{r}^{r}=O\left(|\log \varepsilon| \varepsilon^{\frac{n-p}{p(p-s)} \cdot r}\right) .
$$

If $r<p^{*}\left(1-\frac{1}{p}\right)$, then $-\frac{n-p}{p-1} r+n-1>-1$. We conclude that

$$
\begin{aligned}
\left\|u_{\varepsilon}\right\|_{r}^{r} & =O(1)+\omega_{n} \int_{0}^{R}\left(\varepsilon+x^{\frac{p-s}{p-1}}\right)^{-\frac{n-p}{p-s} r} x^{n-1} d x \\
& \leq O(1)+\omega_{n} \int_{0}^{R} x^{-\frac{n-p}{p-1} r+n-1} d x=O(1)
\end{aligned}
$$

and

$$
\left\|v_{\varepsilon}\right\|_{r}^{r}=O\left(\varepsilon^{\frac{n-p}{p(p-s)} r}\right) .
$$

If $r>p^{*}\left(1-\frac{1}{p}\right)$, then $-\frac{n-p}{p-1} r+n-1<-1$ and $-\frac{n-p}{p-s} r+\frac{p-1}{p-s} n<0$. We have

$$
\begin{aligned}
\left\|u_{\varepsilon}\right\|_{r}^{r} & =O(1)+\omega_{n} \varepsilon^{-\frac{n-p}{p-s} r+\frac{p-1}{p-s} n} \int_{1}^{\infty}\left(1+x^{\frac{p-s}{p-1}}\right)^{-\frac{n-p}{p-s} r} x^{n-1} d x \\
& =O\left(\varepsilon^{-\frac{n-p}{p-s} r+\frac{p-1}{p-s} n}\right),
\end{aligned}
$$

and

$$
\left\|v_{\varepsilon}\right\|_{r}^{r}=O\left(\varepsilon^{-\frac{n-p}{p-s} r+\frac{p-1}{p-s} n+\frac{n-p}{p(p-s)} r}\right)=O\left(\varepsilon^{\frac{p-1}{p-s}\left(n-\frac{r(n-p)}{p}\right)}\right) .
$$

(3), (4) and (5) are thus proved.

For (7) and (8), we have

$$
\begin{aligned}
& \int_{\Omega} \frac{\left|u_{\varepsilon}\right|^{\alpha}}{|x|^{s}} d x=O(1)+\int_{|x| \leq R}\left(\varepsilon+|x|^{\frac{p-s}{p-1}}\right)^{-\frac{n-p}{p-s} \alpha}|x|^{-s} d x \\
& \quad=O(1)+\omega_{n} \int_{0}^{R}\left(\varepsilon+r^{\frac{p-s}{p-1}}\right)^{-\frac{n-p}{p-s} \alpha} r^{-s} \cdot r^{n-1} d r \\
& \quad=O(1)+\omega_{n} \int_{0}^{R \varepsilon^{-\frac{p-1}{p-s}}}\left(1+r^{\frac{p-s}{p-1}}\right)^{-\frac{n-p}{p-s} \alpha} r^{n-s-1} \varepsilon^{-\frac{n-p}{p-s} \alpha+(n-s) \frac{p-1}{p-s}} d r .
\end{aligned}
$$

If $\alpha=q-1$, then $-\frac{n-p}{p-s} \alpha+(n-s) \frac{p-1}{p-s}=-1$. We have

$$
\begin{aligned}
\int_{\Omega} \frac{\left|u_{\varepsilon}\right|^{\alpha}}{|x|^{s}} d x & =O(1)+O\left(\varepsilon^{-1}\right) \omega_{n} \int_{0}^{R \varepsilon^{-\frac{p-1}{p-s}}}\left(1+r^{\frac{p-s}{p-1}}\right)^{-\frac{n-p}{p-s} \alpha} r^{n-s-1} d r \\
& =O(1)+O\left(\varepsilon^{-1}\right) \omega_{n} \int_{0}^{\infty}\left(1+r^{\frac{p-s}{p-1}}\right)^{-\frac{n-p}{p-s} \alpha} r^{n-s-1} d r=O\left(\varepsilon^{-1}\right)
\end{aligned}
$$

since $n-s-\frac{n-p}{p-1}(q-1)=\frac{s-p}{p-1}<0$. Then

$$
\int_{\Omega} \frac{\left|v_{\varepsilon}\right|^{\alpha}}{|x|^{s}} d x=O\left(\varepsilon^{-1}\right) \cdot \varepsilon^{\frac{n-p}{p(p-s)}(q-1)}=O\left(\varepsilon^{\frac{p-1}{p}\left(\frac{n-p}{p-s}\right)}\right) .
$$


If $\alpha=1$, since $-\frac{n-p}{p-1}+n-s>-\frac{n-p}{p-1}+n-p=(n-p)\left(1-\frac{1}{p-1}\right) \geq 0$ for $p \geq 2$, we have

$$
\begin{aligned}
\int_{\Omega} \frac{\left|u_{\varepsilon}\right|}{|x|^{s}} d x & =O(1)+\omega_{n} \int_{0}^{R}\left(\varepsilon+r^{\frac{p-s}{p-1}}\right)^{-\frac{n-p}{p-s}} r^{n-s-1} d r \\
& \leq O(1)+\omega \int_{0}^{R} r^{-\frac{n-p}{p-1}+n-s-1} d r=O(1)
\end{aligned}
$$

and furthermore

$$
\int_{\Omega} \frac{\left|v_{\varepsilon}\right|}{|x|^{s}} d x=O\left(\varepsilon^{\frac{n-p}{p(p-s)}}\right)
$$

(7) and (8) are thus proved.

Note that the above results are well known for the extremal functions associated to the Sobolev embedding, that is, when $s=0$. In the following lemma, we prove additional properties in the case where $s>0$.

Lemma 11.2. For $0 \leq t<p$, we have

$$
\int_{\Omega} \frac{\left|v_{\varepsilon}\right|^{\alpha}}{|x|^{t}}= \begin{cases}O\left(\varepsilon^{\frac{n-p}{p^{2}} \alpha}|\log \varepsilon|\right), & \alpha=\frac{n-t}{n-p}(p-1), \\ O\left(\varepsilon^{\frac{(n-p)(p-1)}{p^{2}}\left(p^{*}(t)-\alpha\right)}\right), & \alpha>\frac{n-t}{n-p}(p-1), \\ O\left(\varepsilon^{\frac{n-p}{p^{2}} \alpha}\right), & \alpha<\frac{n-t}{n-p}(p-1) .\end{cases}
$$

Proof. As above, let $\omega_{n}$ denote the surface area of the $(n-1)$ sphere $S^{n-1}$ in $\mathbf{R}^{n}$. Then

$$
\int_{\Omega} \frac{\left|u_{\varepsilon}\right|^{\alpha}}{|x|^{t}}=O(1)+\omega_{n} \int_{0}^{R}\left(\varepsilon+r^{\frac{p}{p-1}}\right)^{\frac{p-n}{p} \alpha} r^{n-t-1} d r .
$$

Case 1. $\alpha=\frac{n-t}{n-p}(p-1)$ (then $\left.\frac{p-n}{p-1} \alpha+n-t=0\right)$. Then

$$
\begin{aligned}
\int_{\Omega} \frac{\left|u_{\varepsilon}\right|^{\alpha}}{|x|^{t}} & =O(1)+\omega_{n} \int_{0}^{R \varepsilon^{-\frac{p-1}{p}}}\left(1+r^{\frac{p}{p-1}}\right)^{\frac{p-n}{p} \alpha} r^{n-t-1} d r \\
& =O(1)+\omega_{n} \int_{0}^{R \varepsilon^{-\frac{p-1}{p}}} \frac{1}{r} d r=O(|\log \varepsilon|) .
\end{aligned}
$$

Case 2. $\alpha>\frac{n-t}{n-p}(p-1)\left(\right.$ then $\left.\frac{p-n}{p-1} \alpha+n-t-1<-1\right)$. Then

$$
\begin{aligned}
\int_{\Omega} \frac{\left|u_{\varepsilon}\right|^{\alpha}}{|x|^{t}} & =O(1)+\omega_{n} \int_{0}^{R \varepsilon^{-\frac{p-1}{p}}}\left(1+r^{\frac{p}{p-1}}\right)^{\frac{p-n}{p} \alpha} r^{n-t-1} d r \varepsilon^{\frac{p-n}{p} \alpha+(n-s) \frac{p-1}{p}} \\
& =O(1)+O\left(\varepsilon^{\frac{p-n}{p} \alpha+(n-s) \frac{p-1}{p}}\right) .
\end{aligned}
$$

Case 3. $\alpha<\frac{n-t}{n-p}(p-1)$ (i.e. $\left.\frac{p-n}{p-1} \alpha+n-t-1>-1\right)$. Then

$$
\begin{aligned}
\int_{\Omega} \frac{\left|u_{\varepsilon}\right|^{\alpha}}{|x|^{t}} & =O(1)+\omega_{n} \int_{0}^{R}\left(\varepsilon+r^{\frac{p}{p-1}}\right)^{\frac{p-n}{p} \alpha} r^{n-t-1} d r \\
& =O(1)+\omega_{n} \int_{0}^{R} r^{\frac{p-n}{p-1} \alpha+n-t-1} d r \\
& =O(1) .
\end{aligned}
$$

Now, the conclusion follows immediately. 


\section{REFERENCES}

[1] T. Aubin: Nonlinear Analysis on Manifolds. Monge-Ampère Equations, Springer-Verlag, 1982. MR 85j:58002

[2] G. Bliss: An Integral Inequality, J. London Math. Soc., 5 (1930) 40-46.

[3] L. Boccardo and F. Murat: Almost Everywhere Convergence of the Gradients of Solutions to Elliptic and Parabolic Equations, Nonlinear Analysis, TMA 19, (1992) 581-597. MR 93h:35061

[4] H. Brezis, E. Lieb: A Relation Between Point Convergence of Functions and Convergence of Functionals, Proc. Amer. Math. Soc. 88(1983), 486-490. MR 84c:28003

[5] H. Brezis and L. Nirenberg: Positive Solutions of Nonlinear Elliptic Equations involving Critical Exponents, Communications on Pure and Applied Mathematics, 34 (1983), 437-477. MR 84h:35059

[6] H. Brezis and L. Nirenberg: A Minimization Problem with Critical Exponent and Non-zero Data, in "Symmetry in Nature", Scuola Norm. Sup. Pisa (1989), 129-140.

[7] L. Caffarelli, B. Gidas and J. Spruck: Asymptotic Symmetry and Local Behavior of Semilinear Elliptic Equations with Critical Sobolev Growth, Comm. Pure Appl. Math. 42 (1989) 271-477. MR 90c:35075

[8] L. Caffarelli, R. Kohn and L. Nirenberg: First Order Interpolation Inequality with Weights, Compositio Math. 53 (1984) 259-275. MR 86c:46028

[9] G. Cerami, S. Solimini, and M. Struwe: Some Existence Results for Super-linear Elliptic Boundary Value Problems Involving Critical Exponents, J. Functional Analysis 69, 9 (1986) 289-306. MR 88b:35074

[10] K. S. Chou and C. W. Chu: On the Best Constant for a Weighted Sobolev-Hardy Inequality, J. London Math. Soc. (2) 48 (1993) 137-151. MR 94h:46052

[11] P. Drabek: Strongly Nonlinear Degenerated and Singular Elliptic Problems, Pitman Research Notes in Mathematics Series 343 (1996) 112-146. MR 97k:35091]

[12] P. Drabek and S. I. Pohozaev: Positive Solutions for the p-Laplacian: Application of the Fibering Method, Proceeding of the Royal Society of Edinburgh, 127A (1997) 703-726. MR 98h:35061

[13] J. P. Garcia Azorero and I. Peral Alonso: Hardy Inequalities and Some Critical Elliptic and Parabolic Problems, J. of Differential Equations 144 (1998) 441-476. MR 99f:35099

[14] J. Garcia Azorero and I. Peral Alonso: Multiplicity of Solutions for Elliptic Problems with Critical Exponent or with a Non-symmetric Term, Trans. Amer. Math. Soc. 323 (1991), 877-895. MR 99g:35108

[15] J. Garcia Azorero and I. Peral Alonso: Some Results about the Existence of a Second Positive Solution in a Quasi-linear Critical Problem, Indiana University Math. Journal 43 (1994), 941-957. MR 95i:35093

[16] J. Garcia Azorero and I. Peral Alonso: Existence and Non-uniqueness for the p-Laplacian: Nonlinear Eigenvalues. Commun. in PDE, 12 (1987), 1389-1430. MR 89e:35058

[17] N. Ghoussoub: Duality and Perturbation Methods in Critical Point Theory, Cambridge University Press (1993). MR 95a:58021

[18] D. Gilbarg, N. S. Trudinger: Elliptic Partial Differential Equations of Second Order, Springer-Verlag (1983). MR 86c:35035

[19] M. Guedda, L. Veron: Local and Global Properties of Solutions of Quasi-linear Elliptic Equations, Journal of Differential Equations 76 (1988) 159-189. MR 89k:35078

[20] M. Guedda, L. Veron: Quasi-linear Elliptic Equations Involving Critical Sobolev Exponents, Nonlinear Anal TMA, 13 (8) (1989) 879-902. MR 90h:35100

[21] E. H. Lieb: Sharp Constants in the Hardy-Littlewood-Sobolev and Related Inequalities, Ann. of Math. 118 (1983) 349-374. MR 86i:42010

[22] E. H. Lieb and M. Loss: Analysis, Graduate Studies in Mathematics Vol. 14 Amer. Math. Soc., Providence, RI, (1997). MR 98b:00004

[23] M. Otani: Existence and Nonexistence of Nontrivial Solutions of Some Nonlinear Degenerated Elliptic Equations, Journal of Functional Analysis 76 (1988) 140-159. MR 89c:35067

[24] E. S. Noussair, C. A. Swanson, J. Yang: Quasi-linear Elliptic Problems with Critical Exponents, Nonlin. Anal. 20 (1993) 285-301. MR 94b:35106

[25] I. Peral: Multiplicity of Solutions for the p-Laplacian, Lecture Notes at the Second School on Nonlinear Functional Analysis and Applications to Differential Equations at ICTP of Trieste (April 21 - May 9, 1997). 
[26] M. Struwe: Variational Methods. Applications to Nonlinear Partial Differential Equations and Hamiltonian Systems, Second Edition, Springer-Verlag (1996). MR 98f:49002

[27] G. Talenti: Best Constant in Sobolev Inequalities, Ann. Math. Pura Appl. 110 (1976), 353372. MR 57:3846

[28] G. Tarantello: Nodal Solutions of Semi-linear Elliptic Equations with Critical Exponent, Differential and Integral Equations, 5 (1992) 25-42. MR 92k:35109

[29] P. Tolksdorf: Regularity for a More General Class of Quasi-linear Elliptic Equations, Journal of Differential Equations 51 (1984) 126-150. MR 85g:35047

[30] J. Yang: Positive Solutions of Quasi-linear Elliptic Obstacle Problems with Critical Exponents, Nonlinear Analysis, TMA 25 (1995) 1283-1306. MR 96m:35120

[31] C. Yuan: On Non-homogeneous Quasi-linear PDEs Involving the p-Laplacian and the Critical Sobolev Exponent, Ph.D. Dissertation, UBC 1998.

Department of Mathematics, The University of British Columbia, Vancouver, B.C. V6T 1Z2, CANADA

Department of Mathematics, The University of British Columbia, Vancouver, B.C. V6T 1Z2, CANADA 